\title{
Ear as an alternative way for brain drug targeting: An Overview
}

\author{
Sushant Kumar ${ }^{1}$, N.V. Satheesh Madhav ${ }^{2}$ \\ ${ }^{1}$ (Research Scholar, School of Pharmaceutical Sciences, I.F.T.M. University,Moradabad, U.P. , India) \\ ${ }^{2}$ (D.I.T.University, Faculty of Pharmacy, Mussorie Diversion Road Villmakkwalap, Bhagwantpur, Dehradun, \\ India)
}

\begin{abstract}
The brain is a delicate organ, and evolution built very efficient ways to protect it. The same mechanisms that protect it against intrusive chemicals can also frustrate therapeutic interventions. Many existing pharmaceuticals are rendered ineffective in the treatment of cerebral diseases due to our inability to effectively deliver and sustain them within the brain. The article reviews the feasibility of delivering drugs to brain via ear, and provides a novel route for delivering drugs to the brain tissues. The targeted delivery has been shown to reduce the toxicity and increase the therapeutic efficacy many-fold. The potential drugs for the treatment of most brain diseases are therefore often not able to cross these barriers. As a result, various drug delivery and targeting strategies are currently being developed and evaluated to enhance the transport and distribution of drugs into the brain. Ear drug administration in naoparticulate form shows great potential and offers a promising alternative to brain-targeted drug delivery.
\end{abstract}

Keywords: Brain drug targeting, biopolymers, ear physiology, ear to brain connection, nanoparticles

\section{Introduction}

A number of research findings have directed to method for delivery of brain-targeted drugs to the cerebrospinal fluid via the perilymphatic fluid of the inner ear. The system utilizes the passage of cochlear aqueduct as a drug delivery route from the inner ear to the subarachnoid space of the brain. The delivery system includes an otological conduit which enables transfer of drugs from the auditory ear canal to the inner ear and a wearable dispenser for supplying drugs to the otological conduit. An animal study has shown that the inner ear can be used as a drug delivery route to the cerebrospinal fluid. The perilymphatic fluids in the compartment of the inner ear are connected to the cerebrospinal fluid in the subarachnoid space of the brain via the passage of the cochlear aqueduct. It has been shown that in a live animal, there exists a physiological flow from the compartment of the inner ear to the CSF in the subarachnoid space of the brain. A study that investigated the potential use of the inner ear as a delivery route to the CSF has shown that drugs effectively pass from the round-window membrane (RWM) of the inner ear to the CSF following a single trans-tympanic injection.

The researches has provided a minimally invasive drug delivery means for prolonged administration to the CSF via the round-window membrane of the inner ear. A number of inventions has directed to methods and systems for drug delivery to the cerebrospinal fluid (CSF) via inner ear. More specifically, the invention directed to a minimally invasive method for prolonged delivery of brain-targeted therapeutics to the CSF via the perilymphatic fluid of the ear.

The current findings have provided the methods and systems for drug delivery to the cerebrospinal fluid (CSF) via inner ear. More specifically, the invention directed to a minimally invasive method for prolonged delivery of brain-targeted therapeutics to the CSF via the perilymphatic fluid of the inner ear. [1] The perilymphatic fluid inside the inner ear and the cerebrospinal fluid (CSF) surrounding the central nervous system (CNS) are fluidly connected to one another via the passage of the cochlear aqueduct. Animal studies have shown that the perilymphatic fluid physiologically flows from the inner ear to the CSF in the subarachnoid compartment of the brain.

Madhav et.al als confirmed the connection between the ear and brain by using the benzdiazepinenes by administerin through the ear and sleepnes caused in rabbits by this way has confirmed the connection between ear and brain. Currently a number of bionanoparticles formulation are under preparation for such delivery route for targeting to brain for treatment of various brain diseases like epilepsy,tumors etc.

Zhang, X, Chen, G et all.., (2013) developed novel multiple agents loaded poly (D,L-lactide-coglycolide acid) (PLGA) nanoparticles (NPs) and evaluate their potential for brain delivery via inner ear administration. PLGA NPs loaded with salvianolic acid B (Sal B), tanshinone IIA (TS IIA) and panax notoginsenoside (PNS) were prepared by double emulsion/solvent evaporation method. It was observed that optimized NPs displayed satisfactory encapsulation efficiency and desired sustained-release characteristics. NPs following intratympanic administration (IT) in guinea pigs greatly improved drug distribution within the inner ear, cerebrospinal fluid (CSF) and brain tissues compared with intravenous administration (IV). Pharmacodynamic studies demonstrated that NPs following IT markedly inhibited oxidizing reactions and 
protected the brain from cerebral ischemia reperfusion (I/R) injury by upregulating superoxide dismutase (SOD) activity both in serum and brain tissues, simultaneously significantly reducing the levels of malondialdehyde (MDA) and nitric oxide synthase (NOS). Moreover intratympanic delivery did not cause injury of cochlear function by preliminary study on the toxicity. These findings suggested that PLGA NPs-based delivery system via inner ear administration was a promising candidate to brain delivery for the treatment of brain diseases. The article deals with the feasibility of delivering drugs to brain via ear, and provides a novel route for delivering drugs to the brain tissues. Intra-cochlear administration shows great potential and offers a promising alternative to brain-targeted drug delivery.

\section{Introduction}

\section{Ear anatomy and physiology and its connection to brain}

The central nervous system (CNS) is a sanctuary site and is protected by various barriers. These regulate brain homeostasis and the transport of endogenous and exogenous compounds by controlling their selective and specific uptake, efflux, and metabolism in the brain. The previous researches investigates the feasibility of delivering drugs to brain via inner ear, and provides a novel route for delivering drugs to the brain tissuesNanomedicine is a fast evolving field involving nanoparticles or nanostructures for medical applications. Especially in the underdeveloped field of drug delivery to the brain, there are high expectations for the ability of multifunctional nanoparticles (NPs) to cross the blood-brain barrier (BBB). In the present review the challenges nanoparticles face after injection into the body will be summarized. There is a broad range of biological, chemical and physical hurdles for NPs to reach the brainAn animal study has shown that the inner ear can be used as a drug delivery route to the cerebrospinal fluid. The perilymphatic fluids in the compartment of the inner ear are connected to the cerebrospinal fluid in the subarachnoid space of the brain via the passage of the cochlear aqueduct. It has been shown that in a live animal, there exists a physiological flow from the compartment of the inner ear to the CSF in the subarachnoid space of the brain. More specifically, the invention directed to a mimmally invasive method for prolonged delivery of brain-targeted therapeutics to the CSF via the perilymphatic fluid of the inner ear. The perilymphatic fluid inside the inner ear and the cerebrospinal fluid (CSF) surrounding the central nervous system (CNS) are fluidly connected to one another via the passage of the cochlear aqueduct. Animal studies have shown that the perilymphatic fluid physiologically flows from the inner ear to the CSF in the subarachnoid compartment of the brain. It has been reported that labyrinthine perilymph (PL) is derived from both cerebrospinal fluid (CSF) and vascular supply (Zou et al.,2003). A recent study revealed that there was a communication between PL and CSF through the cochlear aqueduct (Chen et al., 2010b). The cochlear aqueduct connects the perilymphatic compartment with the CSF of the cranium and opens into the basal turn of scala tympani. CSF may reach and mix with PL of the scala tympani, though the transfer of fluid between the two spaces may be limited (Hara et al., 1989). Physiologically, this communication may provide a potential pathway for delivering therapeutic agents to specific regions of the brain via inner ear. Our previous studies demonstrated the feasibility of delivering drugs to the CSF and brain via the inner ear (Chen et al., 2010b, 2007). We found that inner ear delivery enabled micro- and macromolecular drugs (dexamethasone and interferon) directly transport to PL, and further transport to the CSF and brain bypassing the bloodlabyrinthine barrier (BLB), which is anatomically and functionally similar to the BBB (Inamura and Salt, 1992).

\section{Anatomy of the Ear}

The temporal bone houses the structure commonly known as the ear. The temporal bone consists of an outer bony structure that is part of the skull and part of the skull base. It meets with several other bones that are part of the skull and the skull base. Within the temporal bone and at its varied boundaries are noted the carotid artery which goes up into the base of the temporal bone, courses through it and exits up at the front of the temporal bone to proceed on to give blood to the central nervous system. The jugular vein which takes the blood back from the brain area exits at the temporal bone lower level and courses through and indents a portion of the temporal bone [see figure 1]. In regard to what is commonly felt to be the ear, there is an outer ear, a middle ear and an inner ear[45]. Taking the outer ear first, we see the pinna or the ear cosmetically speaking, which resides on the lateral temporal bone. It leads to the external auditory canal which is part of the outer ear structure. That canal is skin lined and ends at the 


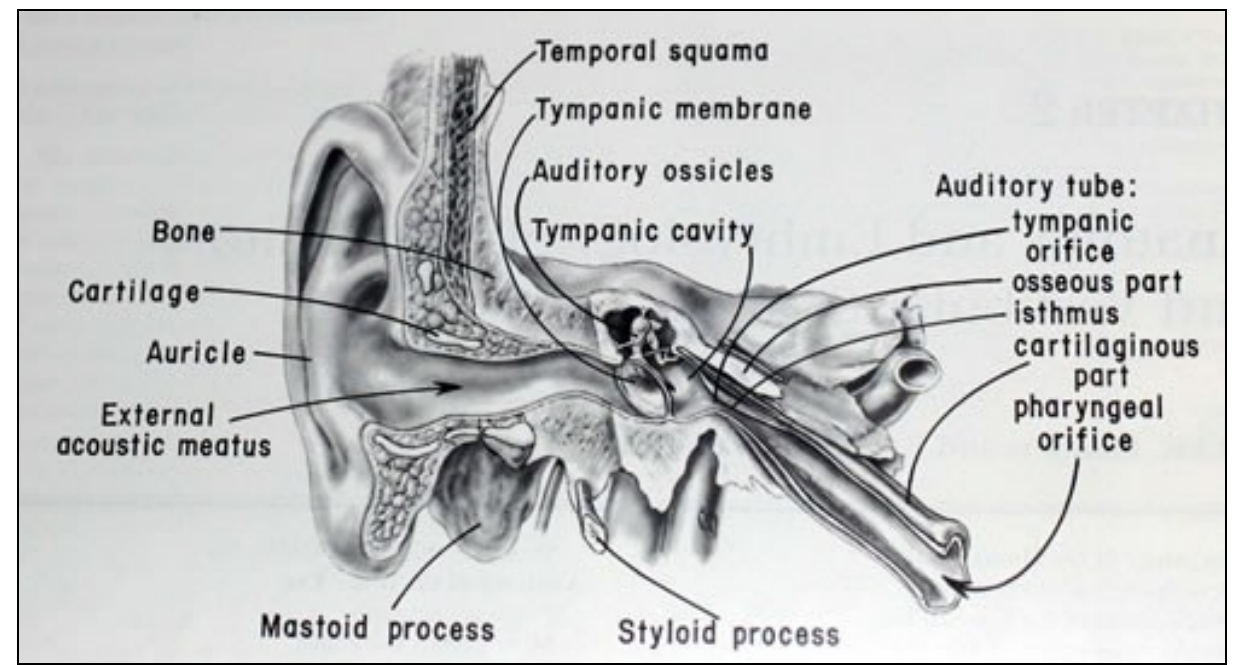

Figure 1. Ear structure

tympanic membrane, or eardrum, the outer surface of which is skin lined. The tympanic membrane has a middle portion, which is fibrous tissue and an inner layer which is mucous membrane, the same sort of mucous membrane, generally speaking, as occurs in the rest of the airway, including the sinuses and the lungs. There are obviously differences among these but they are basically mucous- producing lining cells within the under-surface of the drum in the middle ear. Thus, the outer ear ends at the outer surface of the tympanic membrane. The skin lining should be intact and should stop at that level. The middle ear consists of the inner surface of the tympanic membrane, the surrounding mucous membrane of the air-containing middle ear in which the ossicles or "little bones" of hearing, the malleus, incus and stapes reside. The stapes is the smallest and is the one that seats itself at the junction of the middle ear with the inner ear. The middle ear is an air containing compartment. The inner ear consists of balance organ structures and hearing organ structures. They are in a uni-compartment fluid system. That is to say, it is a self contained unit in which cells change motion activity into neural activity. That occurs on both the vestibular or balance side as well as on the cochlear or hearing side. These structures are known as the labyrinth.

The individual nerves of the inner ear then form into the larger nerves that go through the internal auditory canal (see diagram) and lead directly to the brain. The vast majority of these nerve structures carry the impulses to the brain, a few carry the impulses from the brain out to modulate the inner ear, in particular the vestibular system of the inner ear. There is one more important nerve in this area, which goes through the temporal bone, that is the nerve which moves your face, the facial nerve. This nerve does not give sensation to the face, but gives motion activity to the face. It has a tortuous course that is almost uniformly predictable within the temporal bone. It comes from the brain thorough the internal auditory canal, goes through the middle ear just above the stapes, just under part of the inner ear, the labyrinthine portion called the horizontal semi-circular canal and exits inferiorly out of the temporal bone to gain access to the face. An accessory portion of the aerated middle ear is the mastoid process, which is a honeycomb process of bone that resides basically under the pinna behind the ear canal but is pointed to by most people by pointing behind the ear where the bony prominence is notable. This is variably aerated, it is an area that can become infected and inflamed just as any of these anatomic areas can. Inflammation of this structure is called mastoiditis. There is a nerve for taste which goes through the middle ear without a bony covering which makes it somewhat susceptible to disease processes and to surgical manipulation. Any one of these structures noted above will be referred to during various discussions of the disease processes that can affect the ear. It would be a good idea to refer to this either directly or by downloading a print out of this discussion when you are looking up particular areas of significance in regard to your particular search and your particular disease while perusing the remainder of our web site. The inner ear consists of a membranous "labyrinth" encased in an osseous labyrinth.

The vestibule and semicircular canals are concerned with vestibular function (balance); the cochlea is concerned with hearing. The cochlea is a coiled tube. Notice that the oval window and round window open into the vestibule, at the base of the cochlea. 


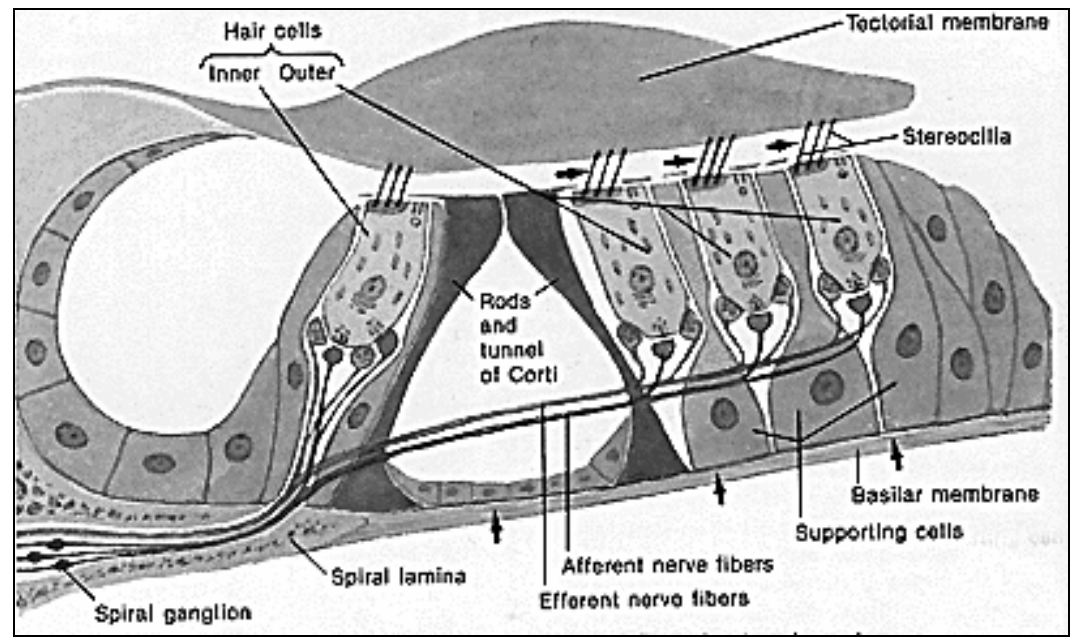

Figure 2. The cochlea

The cochlear coil extends "up" from its base. It is coiled around the modiolus. Reissner's membrane and the basilar membrane divide the the cochlea longitudinally into three scalae. Movement of the the basilar membrane by pressure changes induced by stapes footplate motion at the oval window is a critical step in the transduction process. If you cut the cochlear tube cross sectionally, you'd see something like this Scala vestibuli on top, scala tympani on the bottom [16]. Scala media is a triangular duct in the middle. The process of transduction occurs in the structures within scala media, sitting on the basilar membrane [42], these structures comprise the organ of Corti. The side of the duct where the nerve fibers exit (left in this picture) is the "inner" or "modiolar" side of the duct. The opposite side is the "outer" side. Notice that scala media is more or less triangular, formed by Reissner's membrane, basilar membrane and the structure called the stria vascularis. The fluid that fills scala tympani and scala vestibuli is called perilymph; the fluid that fills scala media is called endolymph. The organ of Corti rests on the basilar membrane within scala media.

Two types of cells in the organ of Corti are support cells and hair cells. The hair cells are the "receptor" cells-- the ones that transduce sound. Support cells such as the Deiter's cells support hair cells. The tops of the hair cells and pillar cells form the reticular lamina, which isolates the hair cells' stereocilia from their cell bodies. The tectorial membrane is loosely coupled to the reticular lamina. Endolymph \& perilymph. There are 4 rows of hair cells, one on the inner (modiolar) side of the tunnel formed by the pillar cells-- these are the inner hair cells; and 3 one the outer side of the Tunnel of Corti, these are the outer hair cells. Notice that the Deiter's cells support the Outer hair cells at their base, but that the outer hair cell walls are surrounded by fluid. The inner hair cell is surrounded by support cells.

The reticular lamina is a solid surface at the tops of the hair cells, so the tops of the hair cells are in endolymph and the bottom of the hair cells are in perilymph. Deiter's cell processes "fill in the gaps" between the tops of the outer hair cells to form the reticular lamina. Outer hair cells, supported by Deiter's cells, form "columns" between the basilar membrane and the reticular lamina.

Stereocilia on inner (left) and outer (right) hair cells [60] [61]. Stereocilia are arranged in curved or vshaped rows that face toward the modiolus.

Each row of stereocilia is taller than the next. The tip of each stereocilium is linked to the side of the stereocilium behind it by a tip link. Nerve fibers exit the organ of Corti on the modiolar side.

In the auditory nerve, the dendrites contact the hair cells. The cell bodies form what is called the spiral ganglion, and the axons form the auditory nerve that connects the ear to the brainstem. The "contact" points between the dendrites and the hair cells or between the axons of one neuron and the dendrites of another are called synapses. Synapses have specialized structures and substances that allow communication between receptors and neurons or between neurons. When a neuron's intracellular electrical potential is changed enough by release of neurotransmitter at a synapse (or in some other way), an abrupt change in electrical potential, an "action potential", occurs. Action potentials are transmitted along the axon to another synapse, where neurotransmitter is released and an action potential may be generated in the neuron on the other side of the synapse (postsynaptic neuron)[15]. The cell bodies of the neurons that form the auditory nerve are located within the cochlear modiolus. The collection of cell bodies is called the spiral ganglion. 


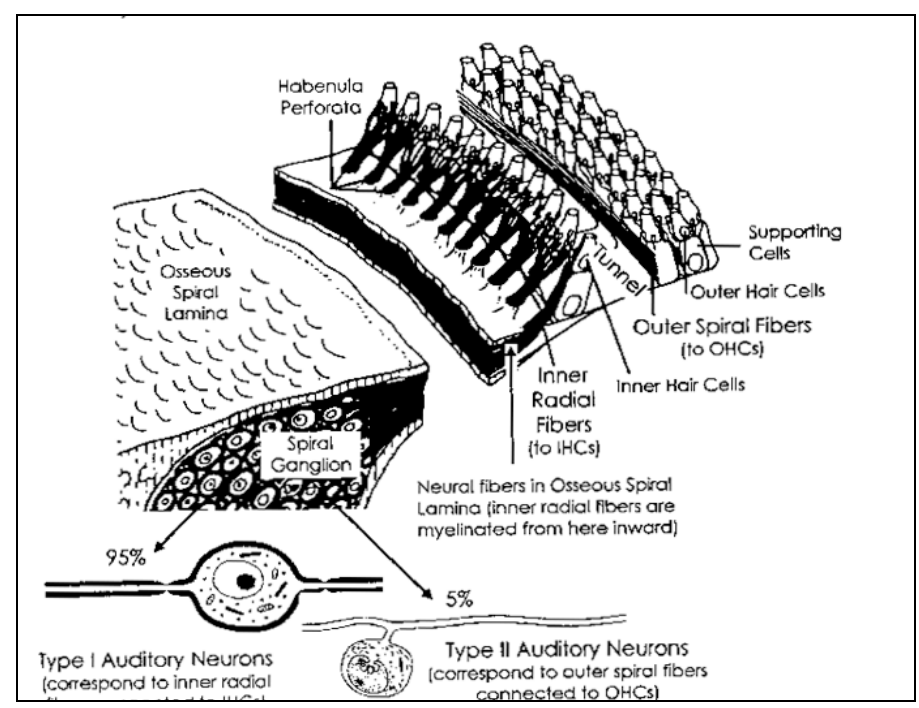

Figure 3. The pattern of afferent innervation

Different types of nerve fibers innervate IHCs and OHCs. Type I fibers innervate IHCs; Type II neurons innervate OHCs. Nearly all of the nerve fibers that carry messages from the ear to the brain innervate inner hair cells. Notice that the many nerve fibers that contact one inner hair cells do not branch to other inner hair cells. Each IHC has its own "private" set of fibers. The Type II nerve fibers innervate many OHCs. and the OHCs they innervate are basal to the point at which the nerve fiber enters the cochlea. Thin fibers attach toward modiolar side, thick fibers toward outer side of IHC. Neurons from the brainstem also contact hair cells. These neurons carry information from the brain to the ear and are called efferent neurons. The vast majority of efferents innervate OHCs, and the contacts on OHCs differ from those on IHCs. Efferents form large calyxshaped contacts on the OHC cell body; efferents form small bouton-like contacts on the afferent nerve fibers that contact IHCs. The nuclei shown here are in a part of the brainstem called the superior olivary complex. Fibers from both sides of the brain innervate both IHCs and OHCs, but the fibers innervating the two types of $\mathrm{HC}$ originate in different places. One recent study suggests that the SOC receives input from auditory cortex[46]. - so fairly high level processing. The fiber tract containing the efferent fibers is known as the olivocochlear bundle (OCB). The tract from the same side of the brain is called the uncrossed OCB and the tract from the opposite side of the brain is called the crossed OCB.

\section{Basic Anatomy and Physiology of the Human Brain}

This chapter contains some basic background on the anatomy and physiology of the human brain relevant to this project. The final section focuses on the neonatal brain and some common pathologies.

\subsection{Anatomy of the head}

The human nervous system consists of the central nervous system (CNS) and peripheral nervous system (PNS). The former consists of the brain and spinal cord, while the latter composes the nerves extending to and from the brain and spinal cord. The primary functions of the nervous system are to monitor, integrate (process) and respond to information inside and outside the body. The brain consists of soft, delicate, nonreplaceable neural tissue. It is supported and protected by the surrounding skin, skull, meninges and cerebrospinal fluid.

\subsubsection{Skin}

The skin constitutes a protective barrier against physical damage of underlying tissues, invasion of hazardous chemical and bacterial substances and, through the activity of its sweat glands and blood vessels, it helps to maintain the body at a constant temperature. Together with the sweat and oil glands, hairs and nails it forms a set of organs called the integumentary system. Figure 2-1 shows a cross-section of the skin and underlying subcutaneous tissue. The skin consists of an outer, protective layer, the epidermis and an inner layer, the dermis. While the top layer of the epidermis, the stratum corneum, consists of dead cells, the dermis is composed of vascularised fibrous connective tissue. The subcutaneous tissue, located underneath the skin, is primarily composed of adipose tissue (fat). 


\subsubsection{Skull}

Depending on their shape, bones are classified as long, short, flat or irregular. Bones of different types contain different proportions of the two types of osseous tissue: compact and spongy bone. While the former has a smooth structure, the latter is composed of small needle-like or flat pieces of bone called trabeculae, which form a network filled with red or yellow bone marrow. Most skull bones are flat and consist of two parallel compact bone surfaces, with a layer of spongy bone sandwiched between. The spongy bone layer of flat bones (the diploë) predominantly contains red bone marrow and hence has a high concentration of blood. The skull is a highly complex structure consisting of 22 bones altogether. These can be divided into two sets, the cranial bones (or cranium) and the facial bones. While the latter form the framework of the face, the cranial bones form the cranial cavity that encloses and protects the brain. All bones of the adult skull are firmly connected by sutures. Figure 2-2 shows the most important bones of the skull. The frontal bone forms the forehead and contains the frontal sinuses, which are air filled cells within the bone. Most superior and lateral aspects of the skull are formed by the parietal bones while the occipital bone forms the posterior aspects. The base of the occipital bone contains the foramen magnum, which is a large hole allowing the inferior part of the brain to connect to the spinal cord. The remaining bones of the cranium are the temporal, sphenoid and ethmoid bones.

\subsubsection{Meninges}

The meninges are three connective tissue membranes enclosing the brain and the spinal cord. Their functions are to protect the CNS and blood vessels, enclose the venous sinuses, retain the cerebrospinal fluid, and form partitions within the skull. The outermost meninx is the dura mater, which encloses the arachnoid mater and the innermost pia mater.

\subsubsection{Cerebrospinal fluid}

Cerebrospinal fluid (CSF) is a watery liquid similar in composition to blood plasma. It is formed in the choroid plexuses and circulates through the ventricles into the subarachnoid space, where it is returned to the dural venous sinuses by the arachnoid villi. The prime purpose of the CSF is to support and cushion the brain and help nourish it. Fig. illustrates the flow of CSF through the central nervous system [16].

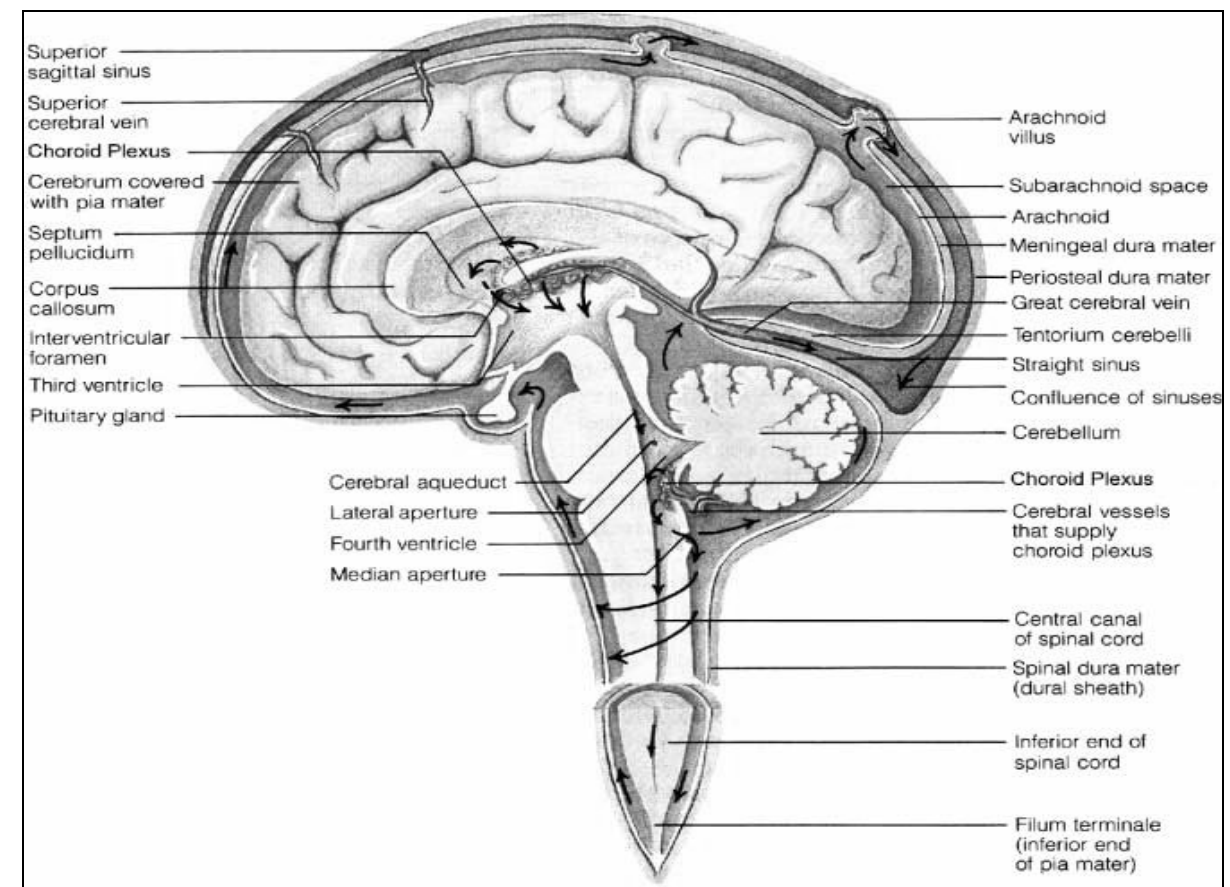

Figure 4. Cerebrospinal Fluid.

\subsubsection{Major regions of the brain and their functions}

The major regions of the brain are the cerebral hemispheres, diencephalon, brain stem and cerebellum.

\subsubsection{Cerebral hemispheres}

The cerebral hemispheres, located on the most superior part of the brain, are separated by the longitudinal fissure. They make up approximately $83 \%$ of total brain mass, and are collectively referred to as the cerebrum. The cerebral cortex constitutes a $2-4 \mathrm{~mm}$ thick grey matter surface layer and, because of its many 
convolutions, accounts for about $40 \%$ of total brain mass. It is responsible for conscious behaviour and contains three different functional areas: the motor areas, sensory areas and association areas. Located internally are the white matter, responsible for communication between cerebral areas and between the cerebral cortex and lower regions of the CNS, as well as the basal nuclei (or basal ganglia), involved in controlling muscular movement.

\subsubsection{Diencephalon}

The diencephalon is located centrally within the forebrain. It consists of the thalamus, hypothalamus and epithalamus, which together enclose the third ventricle. The thalamus acts as a grouping and relay station for sensory inputs ascending to the sensory cortex and association areas. It also mediates motor activities, cortical arousal and memories. The hypothalamus, by controlling the autonomic (involuntary) nervous system, is responsible for maintaining the body's homeostatic balance. Moreover it forms a part of the limbic system, the 'emotional' brain. The epithalamus consists of the pineal gland and the CSFproducing choroid plexus

\subsubsection{Brain stem}

The brain stem is similarly structured as the spinal cord: it consists of grey matter surrounded by white matter fibre tracts. Its major regions are the midbrain, pons and medulla oblongata. The midbrain, which surrounds the cerebral aqueduct, provides fibre pathways between higher and lower brain centres, contains visual and auditory reflex and subcortical motor centres. The pons is mainly a conduction region, but its nuclei also contribute to the regulation of respiration and cranial nerves. The medulla oblongata takes an important role as an autonomic reflex centre involved in maintaining body homeostasis. In particular, nuclei in the medulla regulate respiratory rhythm, heart rate, blood pressure and several cranial nerves. Moreover, it provides conduction pathways between the inferior spinal cord and higher brain centres.

\subsubsection{Cerebellum}

The cerebellum, which is located dorsal to the pons and medulla, accounts for about $11 \%$ of total brain mass. Like the cerebrum, it has a thin outer cortex of grey matter, internal white matter, and small, deeply situated, paired masses (nuclei) of grey matter. The cerebellum processes impulses received from the cerebral motor cortex, various brain stem nuclei and sensory receptors in order to appropriately control skeletal muscle contraction, thus giving smooth, coordinated movements.

\subsection{The cerebral circulatory system}

Blood is transported through the body via a continuous system of blood vessels. Arteries carry oxygenated blood away from the heart into capillaries supplying tissue cells. Veins collect the blood from the capillary bed and carry it back to the heart. The main purpose of blood flow through body tissues is to deliver oxygen and nutrients to and waste from the cells, exchange gas in the lungs, absorb nutrients from the digestive tract, and help forming urine in the kidneys. All the circulation besides the heart and the pulmonary circulation is called the systemic circulation. Since it is the ultimate aim of this research project to image cerebral oxygenation and haemodynamics some aspects of the cerebral circulatory system are described below.

\subsubsection{Blood supply to the brain}

Figure shows an overview of the arterial system supplying the brain. The major arteries are the vertebral and internal carotid arteries. The two posterior and single anterior communicating arteries form the circle of Willis, which equalises blood pressures in the brain's anterior and posterior regions, and protects the brain from damage should one of the arteries become occluded. However, there is little communication between smaller arteries on the brain's surface. Hence occlusion of these arteries usually results in localised tissue damage.

\subsubsection{Cerebral haemodynamics}

The cardiac output is about $5 \mathrm{l} / \mathrm{min}$ of blood for a resting adult. Blood flow to the brain is about $14 \%$ of this, or $700 \mathrm{ml} / \mathrm{min}$. Pressure in the arteries is generated by the heart which pumps blood from its left ventricle into the aorta. (Since pressure was historically measured with a mercury manometer, the units are commonly expressed in terms of [mm Hg], although the official SI unit is the Pascal [Pa].) Hence blood flow is slowest in the small vessels of the capillary bed, thus allowing time for the exchange of nutrients and oxygen to surrounding tissue by diffusion through the capillary walls. Approximately $75 \%$ of total blood volume is 'stored' in the veins which, because of their high capacity, act as reservoirs. Their walls distend and contract in response to the amount of blood available in the circulation. However, the function of cerebral veins, formed from sinuses in the dura mater, is somewhat different from other veins of the body, as they are non-collapsible. 


\subsubsection{Autoregulation}

Panerai 1998 describes autoregulation of blood flow in the cerebral vascular bed as the mechanism by which cerebral blood flow (CBF) tends to remain relatively constant despite changes in cerebral perfusion pressure (CPP). With a constant metabolic demand, changes in CPP or arterial blood pressure that would increase or reduce $\mathrm{CBF}$, are compensated by adjusting the vascular resistance. This maintains a constant $\mathrm{O} 2$ supply and constant CBF. Therefore cerebral autoregulation allows the blood supply to the brain to match its metabolic demand and also to protect cerebral vessels against excessive flow due to arterial hypertension. Cerebral blood flow is autoregulated much better than in almost any other organ. Even for arterial pressure variations between 50 and $150 \mathrm{~mm} \mathrm{Hg}, \mathrm{CBF}$ only changes by a few percent. This can be accomplished because the arterial vessels are typically able to change their diameter about 4-fold, corresponding to a 256-fold change in blood flow. Only when the brain is very active is there an exception to the close matching of blood flow to metabolism, which can rise by up to $30-50 \%$ in the affected areas. It is an aim of PET,

functional MRI, near infrared spectroscopy (NIRS), and, possibly, near infrared imaging, to detect or image such localised changes in cortical activity and associated blood flow.

\subsubsection{Structure and pathologies of the neonatal brain}

Having introduced some basics of the anatomy and physiology of the adult brain, this section focuses on the specific differences in the neonate, as well as common neonatal

Pathologies which have motivated the construction of an instrument capable of imaging cerebral oxygenation, blood volume and, possibly, myelination.

The embryonic brain and spinal cord develop from the neural tube, which is formed by the fourth week of pregnancy. The brain grows immensely in both size and complexity during pregnancy and even soon after birth. Because a membranous skull restricts expansion, the forebrain is bent towards the brain stem, and the cerebral hemispheres almost completely envelop the diencephalon and midbrain

\subsubsection{The blood-brain barrier:}

The blood-brain barrier (BBB) is a dynamic barrier protecting the brain against invading organisms and unwanted substances. It is also the most important barrier impeding drug transport into the brain via the blood circulation. Despite the rapid development in our understanding of the molecular structure of components of the $\mathrm{BBB}[3,4]$, our knowledge in receptor expression at the BBB, advances in medical technology, and breakthroughs in nanotechnology-based approaches, many of the brain or central nervous system (CNS) associated diseases remain under-treated by effective therapies.

Inability of many therapeutic molecules to cross the BBB, the blood-cerebrospinal fluid barrier (BCSFB), or other specialised CNS barriers to reach the specific areas of brain [5] [90] [91]. Such a difficulty in delivering therapeutic molecules to the brain or CNS can only be overcome by a concerted effort in understanding the physiology of BBB, its permeability under different pathological or disease conditions, and its response to physical and chemical stimuli, as well as the various transport receptors at the BBB and available delivery technologies. As many attempts to transport drugs across the BBB could be against the natural function of the BBB, effective approaches or methods should be cautiously assessed with regards to their impact on the overall protective function of BBB. [92] [93].

\subsubsection{Physiology and biology of the blood-brain barrier}

The brain is well protected and dynamically regulated to provide a sanctuary for the central nervous system (CNS). There are several gateways to enter brain parenchyma, the most important two are blood circulation and cerebrospinal fluid (CSF) circulation. In the human brain, there are about 100 billion capillaries in total, providing a combined length of brain capillary endothelium of approximately $650 \mathrm{~km}$ and a total surface area of approximately $20 \mathrm{~m} 2$ [6]. Any molecules' entry into the brain via parenteral administration is strictly controlled by the BBB and the BCSFB. As the surface of BCSFB faces the ventricle that is filled with $\mathrm{CSF}$, not the blood [7], this, in combination with the high turnover rate of CSF, leads to continuously flushing the injected drug (i.e. those injected into the ventricle) back to the blood [8]. The BBB, therefore, is universally considered as the most important barrier in preventing molecules from reaching the brain parenchyma via extensive branches of blood capillary networks. The chief anatomical and functional site of the BBB is the brain endothelium. Physiologically, in addition to brain capillary endothelial cells, extracellular base membrane, adjoining pericytes, astrocytes, and microglia are all integral parts of the BBB supporting system. Together with surrounding neurons, these components form a complex and functional "neurovascular unit" [94] .

A feature of the BBB is its low and selective permeability [95] to molecules which can be attributed to its unique biological characteristics. These include:

1) The lack of fenestrations and with very few pinocytotic vesicles 
2) The presence of tight junctions (TJ) between adjacent endothelial cells, formed by an intricate complex of transmembrane proteins

3) the expression of various transporters including GLUT1 glucose carrier, amino acid carrier LAT1, transferring receptors, insulin receptors, lipoprotein receptors and ATP family of efflux transporters

4) the synergistic inductive functions and up regulating of BBB features by astrocytes, astrocytic perivascular endfeet, pericytes, perivascular macrophages and neurons, as suggested by the strong evidence from cell culture studies [9];

the lack of lymphatic drainage, and absence of major histocompatibility complex (MHC) antigens in CNS with immune reactivity inducible on temporary demand in order to provide maximum protection to neuronal function [10]. All these characteristics lead to BBB to possess multiple functions as a physical barrier (TJ), a transport barrier (P-gp), a metabolic or enzymatic barrier (specialised enzyme systems [11] and an immunological barrier.

\subsubsection{Transport routes across the blood-brain barrier}

It has been well established that there are several transport routes by which solute molecules move across the BBB $[12,22]$. Diffusion of substances into the brain can be divided into paracellular and transcellular. As illustrated in Fig. a, small water-soluble molecules simply diffuse through the TJ but not to any great extent. Small lipid soluble substances like alcohol and steroid hormones penetrate transcellularly by dissolving in their lipid plasma membrane. However, for almost all other substances, including essential materials such as glucose and amino acids, transport proteins (carriers), specific receptor-mediated or vesicular mechanisms (adsorptive transcytosis) are required to pass the BBB [21] [74] [75] [76]. In the case of transport proteins or known as carrier-mediated transport, there is binding of a solute such as glucose or amino acids to a protein transporter[68] [69] [70] on one side of the membrane that triggers a conformational change in the protein, resulting in the transport of the substance to the other side of the membrane, from high to low concentration. If compounds need to be moved against a concentration gradient, ATP may provide the energy to facilitate the process. Efflux pumps or transporters are responsible for extruding drugs from the brain and this mechanism is a major obstacle for the accumulation of a wide range of biologically active molecules in the brain, with the ATP binding cassette (ABC) transporter mechanism of these agents [13] [71] [72] [73]. Inhibition of P-gp in pre-clinical studies has enhanced the penetration of paclitaxel into the brain, indicating the feasibility of achieving improved drug delivery to the brain by suppression of P-gp [14].

\section{Novel approaches for brain targeting}

The field of novel drug delivery has fully emerged and has come into existence as an ideal approach of drug targeting to brain. It mainly includes the use of small colloidal particles. The basic reason of common acceptance of these carriers is due to their controlled profile or drug release nature as well as due to their selected targeting mechanism. Targeting action maybe due to the steric hindrance created by nano-vectors for achieving targeting ability. These carriers are usually administered through parenteral route and due to their steric phenomenon they conceal themselves from opsonisation event induced by tissue macrophages. By this way they achieve targeting ability to brain and other reticuloendothelial system (RES) organs like liver, spleen, etc. Xiaoli Wei et.al. has reviewed that Brain tumor-targeted drug delivery systems, which increase drug accumulation in the tumor region and reduce toxicity in normal brain and peripheral tissue, are a promising new approach to brain tumor treatments. [82] [83] [84] Since brain tumors exhibit many distinctive characteristics relative to tumors growing in peripheral tissues, potential targets based on continuously changing vascular characteristics and the microenvironment can be utilized to facilitate effective brain tumor-targeted drug delivery. [85] [86] [87]

\subsection{Nanoparticles as targeting drug molecules to brain}

From the last few decades nanoparticles have attracted considerable interest in targeting drug molecules [81] to brain [15] [36]. Nano delivery systems have great potential to facilitate the movement of drugs across barriers (e.g., BBB). Nanosystems employed for the development of nano drug delivery systems in the treatment of CNS disorders include polymeric nanoparticles, nanospheres, nanosuspensions, nanoemulsions, nanogels, nano-micelles and nano-liposomes, carbon nanotubes, nanofibers and nanorobots, solid lipid nanoparticles (SLN), nanostructured lipid carriers (NLC) and lipid drug conjugates (LDC) . PEGylated gold nanoparticles are decorated with various amounts of human transferrin (Tf) to give a series of Tf-targeted particles with near-constant size and electrokinetic potential. The effects of Tf content on nanoparticle tumor targeting were investigated in mice bearing s.c. Neuro2A tumors. [88] [89]. In fact, nanotechnology has now emerged as an area of research for invention of newer approaches[70] [78] [79] [80]. The novel properties such as tiny size, tailored surface, better solubility, and multi-functionality of nanoparticles present the capability to interact with composite cellular functions in new ways. The correct mechanism of barrier opening by nanoparticles is not exactly known. But the delivered nanoparticles enter into the brain by crossing the BBB by 
various endocytotic mechVarious drug molecules have been successfully delivered using nanoparticles, e.g., dalargin, kyotorphin, loperamide, tubocurarine and doxorubicin. These peptide based molecules were delivered to brain using polybutylcyanoacrylate nanoparticles coated with poly sorbate-80 (Schronder et al., 1998). Other drugs which are being experimented include clioquinol, thioflavin-T, D-penicillamine, paclitaxel, tacrine, rivastigmine, dexamethasone, 5-fluorouracil, tubocurarine, loperamide. These nanoparticles travel intact and release the drug in brain microenvironment directly which is finally biodegraded due to endocytotic uptake because of very small size by BBB (Gulayaev et al., 1999; Schwarze et al., 1999).

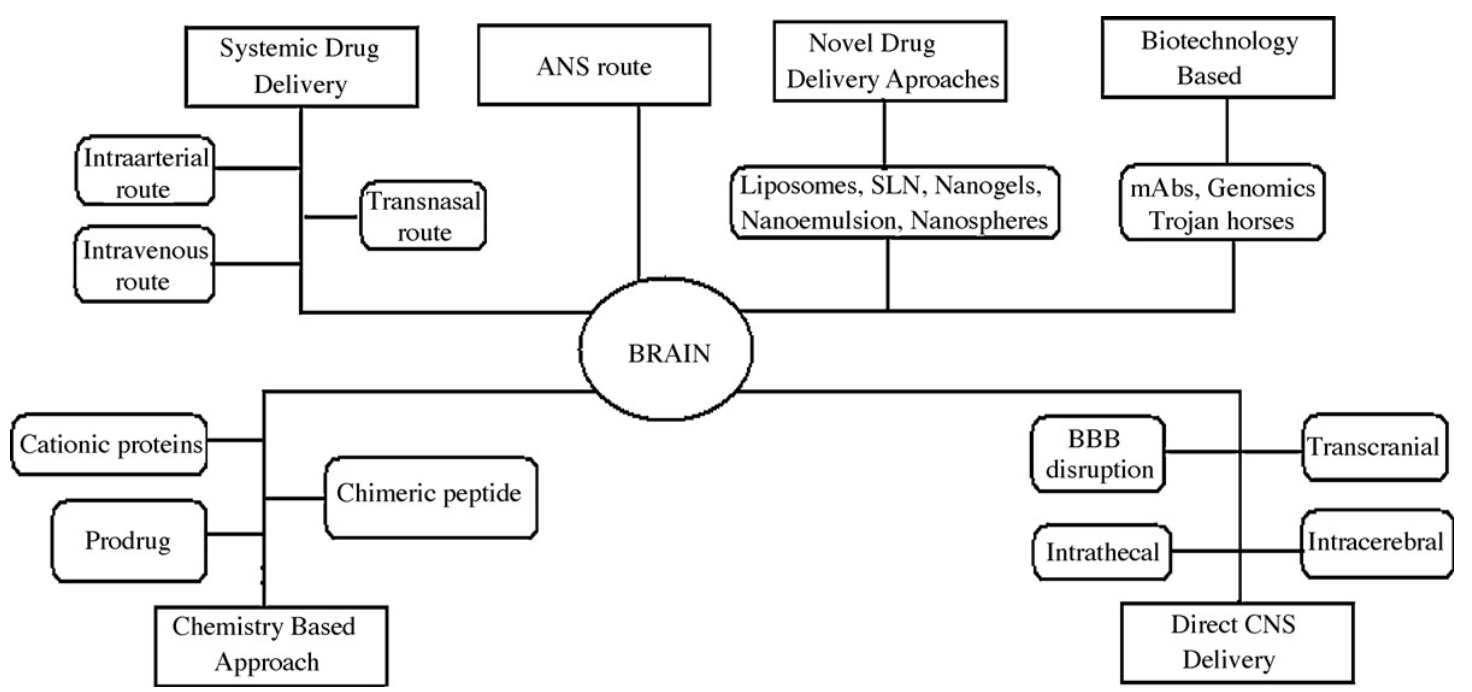

Figure 5. Different approaches of brain targeting

\subsection{Bio-Nanoparticles as current targeting drug carrier molecules by using novel Bio-polymer :}

In present time biopolymer has attracted and concentrated on its use for novel drug delivery and utilization of drug. A number of biopolymers have been isolated by Satheesh Madhav for drug delivery system and most of them are used frequently in various drug delivery systems. A number of biopolymers like Biopolymer from extract of Ocimum basilicum ,Sesamum indicum seeds ,Psidium gaujava Sodium salt of Musa paradisiaca biopolymer,Cocos nucifera , Bombax malabaricum, kernels of Helianthus annus ,fruit pulp of Cordia dichotoma , Mangifera indica ,Lotus corniculatus ,Cajanus indicus ,Logelaria siceraria , Arahcis hypogea seeds ,Lallimantia royalena seeds, Annona squamosa fruit pulp ,Gravia oppositifolia ,Cucurbita maxima fruit pulp ,Psidium guajava , Artocarpus heterophyllus are under identification and isolation. The use of these biopolymers has proved a promising and tremendous use in drug delivery due to its novelistic characteristics and minimised side effectes. These may be used in ear to brain drug targeting for treatment of different brain diseases. Madhav et.al.,(2013) reported the novelistic method for formulating resealed erythrocytes loaded with Repaglinide bionanoparticles. The biopolymer was separated from Citrus sinensis and it was characterized for its physicochemical properties. This invention was also discloses a method for preparing nanoparticles along with resealing of it into goat blood. The formulation showed promising prolongability for 56 hrs apart from this it also showed promising emulsifying ability, retardability and film forming ability which was confirmed by suitable formulations. Conclusion was drawn that this is a novelistic approach significantly delivering the drug for prolonged period and the biopolymer was served as a promising excipient for delivering dosage forms [15]. Madhav \& Priyanka et.al.,(2013) reported the novelistic method for isolating the biopolymer from vigna unguiculata by simplified economical process using anti solvent addition method and it is subjected for various physico chemical properties, spectral analysis like NMR, IR, MASS and morphological studies like SEM and XRD in order to determined its polymeric nature and chemical nature. The novel bio polymer also possesses in built properties like emulsifiability, filmability and retardability which was confirmed by suitably formulating different dosage forms using nimuslide, Zidovudine as a model drug. The conclusion was drawn that this novelistic approach can be adapted for formulating various drug loaded SNEDDS and where as the bio polymer can be used as bio excipient for formulating various drug delivery system [26].

Madhav et.al.,(2013) reported a novel biopolymer from fried seeds of Amaranthus Spinosus. The biopolymer was isolated by simplified economical process using dimethyl ketone as a non solvent. The biopolymer contains a alkane group, alcohlic group and alkenes as a functional group.It also discloses a novel method for preparing biofilm loaded with This biopolymer also possess enhanced property like bioretardent, emulsifying and filmability which confirmed by suitable formulated drug delivery system. So biopolymer can be served as a bioexipient for formulating various drug loaded dosage forms [27]. Madhav et.al.,(2013) reported This invention explores a novelistic composition for formulating Montelukast bio-microcapsules using a novel 
biomaterial which was isolated from fruit pulp of Opuntia aciculata. Biopolymer comprises of prominent mucoadhesive groups (Alcohols, Ethers, Ketones, Alkanes) which was confirmed by IR spectra. Microcapsules were formulated by using biomaterial as a wall material cum retardant and the co-processing agent by solvent evaporation method. The formulated microcapsules showed promising in-vitro and in-vivo drug release for a prolonged time of $24 \mathrm{hrs}$ with good mucoadhesivity. The bio-polymer was screened for other in-built properties like emusifiability, filmability and bio-retardability which were confirmed by suitably formulating drug loaded formulations emulsion, films, microcapulse, nanoparticles and sustained release tablets. The conclusion was drawn that the novel microcapsules showed prolonged drug release and isolated biopolymer displayed in-built properties [28]. Madhav et.al.,(2013) reported This current invention explores a novalistic method for isolating biopolymer from the dry fruit of Pistachio vera. This biomaterial comprises alkane, alkene, ester and aromatic functional groups which were confirmed by IR spectra. It is devoid of toxicity which was confirmed by acute toxicity study. This invention also explores a method of preparing a novel bio nanosuspension loaded with Moxifloxacin and biomaterial as a retardant cum stabilizer. This study revealed that biomaterial showed its inbuilt retardability and stability propreties apart from that it also possess novel in-built properties like emulsifiability, suspendibility, filmability and retardability which was confirmed by suitably formulating drug loaded dosage forms. The conclusion was drawn that bio-material; possess potent inbuilt properties and it can serve as a bio-excipient for formulating various dosage forms [29]. Madhav et.al.,.(2013) reported the method for isolating the biopolymer from fruit pulp of Ficus carica by non-solvent addition method. The biopolymeric material comprises of alkane, alcohol and ether functional groups. It displayed inbuilt properties like emulsifiability, suspensibility, filmability and retardability which were confirmed by suitably formulating drug loaded delivery system. This invention also discloses formulating the bio-microemulsion for ocular drug delivery loaded with Acetazolamide. So biopolymer possesses an inbuilt novelistic properties and it can serve as bio-excipient for delivering various dosage form [30]. Madhav et.al.,(2013) reported In recent years, biodegradable polymer materials (known as biocomposites) have gained particular interest specially in Pharma field. Bio-polymers are continually being employed in an expanding range of areas. As a result, many researchers are keen to modify natural materials to make them more user-friendly, and into designing novel polymer composites out of naturally occurring materials. It is suggested that biodegradable polymer materials will reduce the need for synthetic polymer production at a low cost, thereby producing a positive effect both environmentally and economically. We current expose a method for formulating bioniosomes using a biosurfactant which was isolated from santalum album wood. The biopolymer revealed its inbuilt functional groups containing $\mathrm{NH} 2, \mathrm{CH} 2-, \mathrm{CH} 3-, \mathrm{CN}-$ and $\mathrm{CH} 2-\mathrm{CH}=0$ as a functional groups. The niosomes were formulated by modified thin film hydration andsonication method. Conclusion was drawn that bioniosomes can be used for effectively targeting the drug to the hair follicles and niosomes can be prepared by a novel bioexcipient from santalum album and the biopolymer can also serve as a bio-excipient for formulating various drug delivery dosage forms. This present paper is intended to provide a novel method for extraction of the biomaterial from natural source \& employ it for various pharmaceutical purpose [31]. Madhav et.al.,(2013) reported This invention discloses the method for formulating Emtricitabine Solid lipid Nanoparticles by using bio retardant which was isolated from Mangifera Indica seeds. The isolated bio retardant is devoid of toxicity. Emtricitabine Solid Lipid nanoparticles were formulated by using bio retardant, PVA, Chloroform and other co processing agents by hot melt solvent evaporation method. The formulation showed nanosizing in the range of 442 to $500 \mathrm{~nm}$ with a promising stability and prolonged ability for a period of $24 \mathrm{hrs}$. The isolated bio retardant also possess a novelistic inbuilt retardability, Emulsifiability and Filmability which was confirmed by formulating suitable doses forms using the bio retardant and other co processing agents. Conclusion was drawn that the bio retardant can serve as a promising excipient for formulating controlled or prolonged drug delivery system [32].

\section{Anatomical and physiological connection between ear and brain 5.1. Cochlear blood supply}

\subsubsection{Arterial blood supply}

The arterial blood supply to the cochlea was first described in Europe, most notably in Germ Siebenmann in 1894, and in Japan by Nabeya . Other studies have provided detailed information on the gross and microscopic characteristics of cochlear arteries. The cochlea and the vestibule are supplied by arteries from the same source, namely, the internal auditory artery (labyrinthine artery or arteriae labyrinthi). The internal auditory artery usually arises from the middle cerebral artery (arteria cerebelli inferior anterior), a branch of the basilar artery (arteria basilaris); in some individuals, it arises directly from the basilar artery. There may be two internal auditory arteries; indeed, this variant was found in nearly half the individuals included in an autopsy study. The internal auditory artery arises from the meatal loop of the middle cerebral artery, which is consistently present and penetrates more or less deeply within the internal acoustic meatus. The meatal loop usually sits on the cochlear nerve and is often sandwiched between this nerve and the facial nerve. It also gives 
off the subarcuate artery (arteria subarcuata), which runs in the petromastoid canal, passing through the arch of the superior semicircular canal. The subarcuate artery does not give any branches to the labyrinth; in some individuals, however, the meatal loop gives off a trunk that divides into the subarcuate artery and internal auditory artery. Furthermore, the subarcuate artery may supply branches to the cerebellum.

Therefore, every effort should be made to preserve the proximal subarcuate artery when removing pontocerebellar tumors, in particular to increase the changes of hearing preservation. In contrast, the subarcuate artery can be safely clipped or coagulated posterior to the porus acusticus internus, at the point of entry into the petromastoid canal. The internal auditory artery usually arises from the apex of the meatal loop. It runs along the upper aspect of the cochlear nerve toward the fundus of the internal acoustic meatus. In about $10 \%$ of individuals, it sits on the floor of the internal acoustic meatus. The portion of the internal auditory artery located within the meatus gives off several branches, which have been described in detail. The first branch is the anterior vestibular artery (arteria vestibulare anterior), which supplies the posterior and lateral semicircular canals, the utricle, and the posterior part of the saccule. The cochlea is supplied by the spiral modiolar artery (arteria spiralis modioli) and ve s t i bu 1 o c o chlear art e ry (arteria vestibulo-cochlearis), which arise from the common cochlear artery (arteria cochlearis). The common cochlear artery stems from the internal auditory artery near the site where the cochlear nerve penetrates into the modiolus; it runs through the modiolus and supplies the apex of the cochlea, the second turn, and part of the basal turn. The vestibulocochlear artery arises after the spiral modiolar artery and travels to the vestibule, where it gives off a vestibular branch and a cochlear branch. The vestibular branch supplies the posterior semicircular canal and the saccule, whereas the cochlear branch feeds the proximal part of the base of the cochlea. Figure shows the distribution of the main branches of the internal auditory artery.

As pointed out by Tange, this distribution suggests that the clinical features may vary according to the site of arterial obstruction. Thus, obstruction of the spiral modiolar artery would be expected to cause hearing loss predominating in the low frequencies and obstruction of the vestibulocochlear artery [34] hearing loss predominating in the high frequencies and accompanied with vertigo. In practice, the balance between these two arteries probably varies across individuals, and anastomoses between the two arteries may exist. When removing a neurinoma from the auditory nerve, the various arterial branches supplying the cochlea cannot be identified at the fundus. The internal auditory artery, in contrast, is usually identified during microsurgical dissection. This artery has a muscular media and can therefore develop spasm responsible for cochlear dysfunction .

Therefore, during microsurgical dissection of neurinoma of the auditory nerve, in addition to preserving the cochlear nerve and vessels, surgeons should take care to prevent arterial spasm. Within the cochlea, both spiral and radial vessels are found. The arteries are terminal, forming no anastomoses. They have been described in detail in several animal species and in humans. Large arteries penetrate into the cochlea via the modiolus. The spiral modiolar artery gives off radial branches to the lateral cochlear wall, including the stria vascularis. As the arteries decrease in size, they lose their muscular layer, so that spasm necessarily causes extensive cochlear ischemia. The capillary network in the stria vascularis is extremely rich at the base of the cochlea, compared to the apex. The physiological and pathological impact of this difference in capillary abundance is unclear. The key role played by the stria vascularis in ensuring proper function of the OHC feedback loop suggests that this loop may be essential to the perception of high-frequency sounds but may be less important for low-pitched sounds. The stria vascularis consists roughly of three cell layers: the basal layer facing the perilymphatic space, the intermediate layer, and the marginal layer facing the endolymphatic space. The basal cells are held together by tight junctions that make the stria vascularis impermeable to perilymph. Similarly, the intrastrial space is sealed away from the endolymph by tight junctions linking the marginal cells. The stria vascularis is the only structure in the body where blood vessels are isolated by completely leak-proof cell layers. Howeve $\mathrm{r}$, cross -1 ayer communication occurs via gap junctions, which allow nutrients and metabolites to travel from the perilymph. The endolymph is probably secreted from the perilymph rather than from blood. Secretion of potassium into the endolymph is ensured primarily by energy dependent ion pumps coupled to ATPases.

Cochlear ischemia stops ion pump function nearly instantaneously, inducing a drop in the endolymphatic potential and thereby causing hearing loss. This effect on endolymphatic potential, which is reliably demonstrated by the probe microphone method, occurs within a few seconds of cochlear blood flow arrest. This exquisite sensitivity of the cochlea to ischemia was reported as early as 1961, by Konishi et al.. The ion pumps in the stria vascularis are also extremely sensitive to loop diuretics. Furosemide overdose impairs the $\mathrm{OHC}$ feedback loop as rapidly as does ischemia and therefore causes cochlear dysfunction.

\subsubsection{Cochlear veins}

Venous drainage of the cochlea occurs via the modiolus. Most mammals have a spiral modiolar vein (vena spiralis modioli). In contrast, no main vein is visible among the nerves in the internal acoustic meatus. The 
venous blood empties either directly into the inferiorpetrosal sinus (sinus petrosus inferior) or internal jugular vein (vena jugularis internae) or travels through other venous sinuses via the vein of the vestibular or cochlear aqueduct (vena canales endolymphaticus/ perilymphaticus). The multiplicity of venous drainage channels pro $b$ ably explains why resection of the internal jugular vein or sigmoid sinus (e.g., during surgery for jugular paraganglioma) does not cause hearing impairment [48].

\subsection{Cochlear lymphatics}

Little is known about the cochlear lymphatics, which were long confused with the endo- and perilymphatic system . Lymphoma can arise in the internal acoustic meatus, indicating that lymphoid tissue is present at this site.

\subsection{Cochlear blood flow measurement}

A tool capable of detecting ischemia-induced hearing loss would be extremely useful in clinical practice. Such a tool would allow physicians to use, and to monitor the effectiveness of, treatments specifically targeted at organ ischemia. In patients with sudden hearing loss, [49] [50] [51] [52] for instance, the distinction between ischemic and nonischemic causes is usually impossible at present. The only exception is neurinoma of the auditory nerve: if the tumor is known, the presence of cochlear ischemia can be inferred from the pattern of otoacoustic emissions. Laser Doppler velocimetry is the least invasive method for measuring cochlear blood flow. This method consists in using the Doppler effect to study the speed of a reflected red laser beam directed at the tissue under study. In a given region, higher blood flow is associated with faster blood movement, so that the beam reflected by the erythrocytes shifts toward the blue part of the color spectrum. After calibration, the diffe rences in cochlear blood flow compared to the normal pattern can be determined. Laser Doppler velocimetry allows ready measurement of cochlear blood flow in animal species whose otic capsule opposite the stria vascularis is thin and therefore causes little attenuation of the incident and reflected laser beams. In the Mongolian gerbil, for instance, the cochlear bone is so thin that the stria vascularis can be glimpsed through the otic capsule under the microscope. As a re s u $1 \mathrm{t}$, cochlear blood flow measurement is highly reliable in this species. When the bone is thick, considerable laser beam attenuation occurs. To overcome this problem, the laser Doppler probe can be modified according to bone thickness . A far simpler solution, however, consists in placing the probe opposite the round window so that the laser beams do not need to travel through the bone. This method has proved remarkably effective in rabbits but remains to be studied in humans [1].

\subsection{Fluid Compartments and their distribution}

Most of the structures of the cochlea are protected from the systemic circulation by the presence of a blood-cochlear barrier [38] (or blood-labyrinthine barrier), similar to the blood-brain barrier. The extracellular fluid spaces of the ear can be considered pharmacologically as comprising four components: (1) the systemic circulation-largely confined to the stria vascularis and to fluid contained within the blood vessels and capillaries; (2) perilymph, a fluid similar in composition to CSF that provides a favorable milieu for sensory cell and neuronal function; (3) endolymph, a fluid high in potassium ions that provides the electrochemical drive for sensory cell function; and (4) the extracellular fluid of cochlear bone, which seems to be a transitional region between the systemic circulation and the perilymph.

A small portion of the inner ear [39] [40], the stria vascularis, is readily accessible from the systemic circulation. It is surrounded by cells connected with tight junctions that provide a part of the blood-cochlear barrier [47]. Drugs that act in the stria vascularis can be administered systemically, and the most likely action is a reduction of the endocochlear potential. Because the endocochlear potential is the battery that drives the cochlear transduction process, a reduction in the endocochlear potential will decrease hearing capability. The vascularization of the rest of the cochlea is relatively sparse, although the modiolus and walls of the scala tympani are exceptions. The endothelial cells that line the vasculature are connected with tight junctions and no fenestrations, and this network of tightly coupled endothelial cells is the dominant component of the bloodcochlear barrier. Perilymph, the primary fluid of the inner ear, is in diffusional continuity with CSF via the cochlear aqueduct in most animals, although the transfer of fluid between the two spaces is limited. In humans, the aqueduct is usually not patent, although diffusional continuity is achieved via other pathways, most notably the spaces around and within the auditory nerve.

Perilymph is primarily produced locally via the vascular supply to the cochlea (reviewed in, although a minor contribution from the CSF is possible. The site of perilymph production is not known, although the vascular supply to the modiolus and walls of the scala tympani have been suggested. Perilymph resembles CSF in composition, and this similarity likely arises from a similar mode of production of perilymph and CSF. Endolymph, the highly specialized extracellular fluid that fills the scala media, bathes the apical surfaces of the sensory cells. 
Endolymph is produced primarily in the stria vascularis and is secreted by the marginal cells of the stria vascularis that face the endolymph. Endolymph contains high concentrations of potassium, low concentrations of sodium, and very low concentrations of calcium. The scala media is lined with cells that contain tight junctions. The tight junctions help the scala media retain its unusual electrical potential - around $+100 \mathrm{mV}$, called the endocochlear potential. The endocochlear potential and the high potassium concentration of the scala media provide the electrochemical drive to the hair cells to allow their transduction of mechanical motion. The cochlea is surrounded by petrous bone. This bone normally does not remodel and is very poorly vascularized. The bone is accessible to perilymph via lacuna canaliculi, which are canals or holes in the bone in free communication with the scala tympani. However, the bone is also accessible to the systemic circulation via fenestrated blood vessels. The bone has not been characterized with regard to its presence in or out of the bloodcochlear barrier.

\subsection{Vestibulocochlear Nerve:}

The vestibulocochlear nerve is a sensory nerve that conducts two special senses: hearing (audition) and balance (vestibular). The individual nerves of the inner ear then form into the larger nerves that go through the internal auditory canal (see diagram) and lead directly to the brain. The vast majority of these nerve structures carry the impulses to the brain, a few carry the impulses from the brain out to modulate the inner ear, in particular the vestibular system of the inner ear. There is one more important nerve in this area, which goes through the temporal bone, that is the nerve which moves your face, the facial nerve. The receptor cells for these special senses are located in the membranous labyrinth which is embedded in the petrous part of the temporal bone. There are two specialized organs in the bony labyrinth, the cochlea and the vestibular apparatus. The cochlear duct is the organ that is connected to the three bony ossicles which transduce sound waves into fluid movement in the cochlea. This ultimately causes movement of hair cells which activate the auditory part of the vestibulocochlear nerve. The vestibular apparatus is the organ that senses head position changes relative to gravity. Movement causes fluid vibration resulting in hair cell displacement that activates the vestibular part of the eighth nerve. The peripheral parts of the eighth nerve travel a short distance to nerve cell bodies at the base of the corresponding sense organs. From these peripheral sensory nerve cells the central part of the nerve then travels through the internal auditory meatus with the facial nerve. The eighth nerve enters the brain stem at the junction of the pons and medulla lateral to the facial nerve. The auditory component of the eighth nerve terminates in a sensory nucleus called the cochlear nucleus which is located at the junction of the pons and medulla. The vestibular part of the eight nerve ends in the vestibular nuclear complex located in the floor of the fourth ventricle.

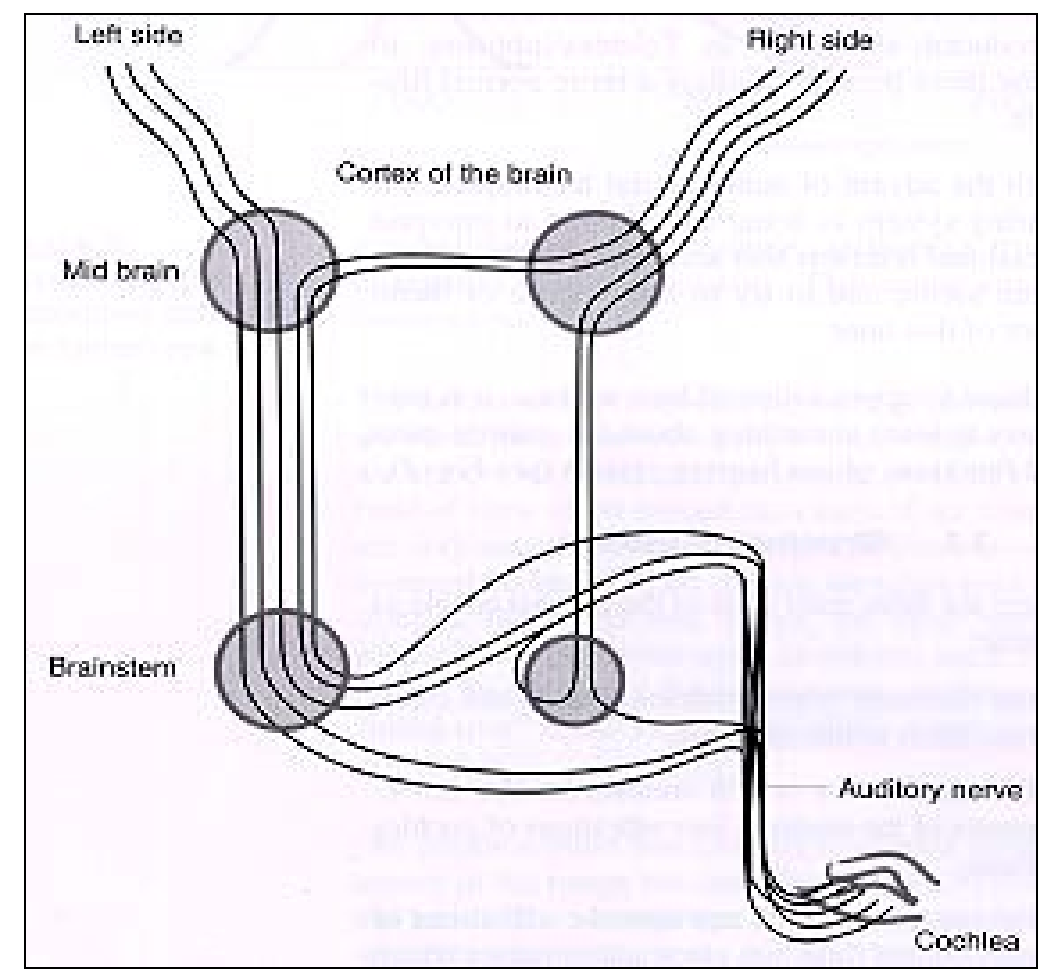

Figure 6. Auditory nerve towards the cortex of the brain 
Nerve impulses are transmitted from the ear to the brain via the auditory nerves, one of the several sensory nerves that exists in the group of nerves known as cranial nerves. The auditory nerves connect the nerve impulses of the ears to the upper "temporal lobe" of the "cerebral cortex". Nerve impulses pass over "neurons" via an electro-chemical action. That is, the neuron itself provides the necessary energy to propel the impulse along the nerve. The nerve impulse does not travel as fast as a standard electrical current, but instead moves at about 3.25 to 395 feet/sec. Nerve impulses travel over many neurons on their way to the brain. Neurons work together by transmitting the impulses through the "axons" to the "dendrites" of the neuron. The dendrites of one neuron communicate to the dendrites of another by means of the "synapse". This gap-like structure communicates by releasing a chemical transmitter substance.

\subsection{Drug Distribution}

Once a drug enters the cochlear fluids, [35] [37] its distribution is governed by many of the same pharmacokinetic variables seen in the bloodstream. Most of the structures of the cochlea are in diffusional continuity with the perilymph [16]. Thus, a drug placed into the perilymph can diffuse into the organ of Corti, which contains hair cells, neuronal terminals, and other specialized cells. The perilymph is also in diffusional continuity with the spiral ganglion and structures within the modiolus of the cochlea. Access to structures in the modiolus is enhanced by the presence of numerous lacuna canaliculi on the surface of the osseous spiral lamina . These perforations are on the order of 0.2 to $23 \mu \mathrm{m}$ in diameter. Finally, the spiral ligament and spiral limbus are also in diffusional continuity with perilymph. Cochlear structures [33] that are not accessible from the perilymph include the stria vascularis and the scala media, and thus the surfaces of cells that line the scala media. Both structures are lined with cells that are connected via tight junctions. The protein composition of perilymph in the human has a number of elements in common with that of blood, although perilymph concentrations are lower, and protein interactions with drugs are as important in the perilymph as in blood. Albumin levels are high and can bind acidic drugs, and acid glycoproteins can bind basic drugs. Partition coefficients of drugs with these proteins will determine free concentration of the drug. These proteins can provide a reservoir for accumulation of the drug in perilymph over time.

Idiopathic sensorineural hearing loss (ISSHL) [53] [54] [55] [56]is a sudden decline in hearing affecting 3 or more subsequent frequencies by at least $30 \mathrm{~dB}$ developing over 3 days or less, without identifiable cause. Administration of oral st eroids is widely used with well known effectiveness. Recently, treatment with intratympanic injections (ITI) of steroids have been suggested, however hard evidence of its superiority is lacking. This study retrospectively reviewed the success rate of ITI both as initial and as salvage therapy. Severe to profound deafness is most often secondary to a loss of or injury to cochlear mechanosensory cells, and there is often an associated loss of the peripheral auditory neural structures, specifically the spiral ganglion neurons and peripheral auditory fibers. Cochlear implantation is currently our best hearing rehabilitation strategy for severe to profound deafness. These implants work by directly electrically stimulating the remnant auditory neural structures within the deafened cochlea. When administered to the deafened cochlea in animal models, neurotrophins, [57] [62] [63] [64] [65] [67] specifically brain derived neurotrophic factor and neurotrophin-3, have been shown to dramatically improve spiral ganglion neuron survival and stimulate peripheral auditory fiber regrowth. In animal models, neurotrophins administered in combination with cochlear implantation has resulted in significant improvements in the electrophysiological and psychophysical measures of outcome.

The distribution of drugs within the inner ear is dependent on the point of entry of the agents. The immune system can attack just the ear, attack the ear and some other body part like the eye, or attack the entire body (including the ear). An autoimmune [57] [58] [59] reaction also creates debris. Even if the ear is not being directly attacked, it can end up with debris transported from distant locations and deposited by the circulation. This debris in the ear can cause problems.

There are three practical entry points: through or near the RWM (at the base of the cochlea on the scala tympani side), through or near the oval window (at the base of the cochlear on the scala vestibuli side), or through the bone of the cochlea via application in the middle ear. The cochlea is a long set of coiled tubes. The perilymph of the scala tympani can be thought of as beginning at the base of the cochlea near the RWM, continuing apically to the end of the tube where it then connects with the perilymph of the scala vestibuli and continues back down to the base to the oval window where the footplate of the stapes is located. Drug transport via simple diffusion along the length of the perilymphatic space, because of the large length relative to cross section, would require very long times (hours to days) for significant drug distribution throughout the cochlea. Salt, Plontke, and colleagues have modelled the distribution of drugs applied at the RWM extensively. They suggest that in addition to diffusion along the length of the cochlea, diffusion through the tissue of the cochlea from one scala to another must be considered as we. The magnitude of the interscalar distribution will be highly dependent on the drug. Highly lipid soluble drugs like steroids will have a greater component of their distribution through tissue than would a drug with a high rate of cellular uptake via carrier systems. Many drugs 
are delivered into the middle ear space with the hope that they will diffuse through the RWM into the scala tympani of the basal turn. The round window is an Achilles heel in the blood-cochlear barrier. It is permeable to water and many drugs. The major problems with this mode of entry from a pharmacokinetic perspective are that it relies on simple diffusion to pass drug from the middle to the inner ear, and once in the scala tympani, the only mode of distribution is simple diffusion in a fluid space with little turnover. Using implanted electrodes and tracer ions, the longitudinal volume flow in the scala tympani was determined to be negligible, approximately $1.6 \mathrm{~nL} / \mathrm{min}$ directed apically. The RWM can vary greatly in thickness, which can lead to variability in absorption of drug into the scala tympani. Delivery of agent into the scala vestibuli does not provide as favorable access to the structures of the organ of Corti as application via the scala tympani. In theory, access to the auditory neurons should be possible via canal perforantes in the scala vestibuli portion of the osseous spiral lamina. Also, there are possible diffusion pathways to the scala tympani via entry into and exit from the modiolus as well as into and out of the spiral ligament [2].

\section{Ear as an alternative route for brain drug targetting}

Ivri, Yehuda (2010) invention deals with methods [17] and systems for drug delivery to the cerebrospinal fluid (CSF) via inner ear. More specifically, the invention directed to a minimally invasive method for prolonged delivery of brain-targeted therapeutics to the CSF via the perilymphatic fluid of the inner ear.[23,24] The perilymphatic fluid inside the inner ear and the cerebrospinal fluid (CSF) surrounding the central nervous system (CNS) are fluidly connected to one another via the passage of the cochlear aqueduct. Animal studies have shown that the perilymphatic fluid physiologically flows from the inner ear to the CSF in the subarachnoid compartment of the brain. An animal study that investigated the feasibly of using the inner ear as a delivery route to the CSF has shown that dexamethason acetate loaded to solid-lipid nanoparticles (SLN) effectively transports to the CSF following trans-tympanic injection[16]. Shulman A et. al. reported that in all traumatic brain injury (TBI) patients with a clinical history of closed or penetrating head injury, the initial head trauma is associated with a vibratory sensation and noise exposure, with resultant alteration in vascular supply to the structures and contents of the fluid compartments of brain and ear[12] (i.e., the fluid dynamics vascular theory of brain-inner-ear function [FDVTBE]). The primary etiology-head trauma-results in an initial fluctuation, interference, or interaction in the normal fluid dynamics between brain and labyrinth of the inner ear, with a resultant clinical diversity of complaints varying in time of onset and severity. Normal function of the brain and ear is a reflection of a normal state of homeostasis between the fluid compartments in the brain of cerebrospinal fluid and perilymph-endolymph in the labyrinth of the ear [18].

Chen G. et al., (2007) reported that dexamethason reaches the forth ventricle compartment of the brain following administration via the inner ear [20]. Due to dynamic distribution and bulk flow within the subarachnoid space, CSF that is produced in ventricular system flows from the forth ventricle directly into the cisterna magna. The study on brain-targeted drug delivery via inner ear has done (Chen G. et al [2007]). Importantly, samples for the pharmacokinetic study described therein were extracted from the fourth ventricle of the brain [7]. This indicates that drugs delivered via the inner ear are distributed in the brain's fourth ventricle and suggests that the delivery via the inner ear may be used in targeting the tissue of the brain [8] inside and at the vicinity of the fourth ventricle. One such therapeutic application is in treatment of pain. The floor of the fourth ventricle has been implicated in the control of nociceptive transmission at the spinal level. Delivery of drugs to the CSF via the inner ear was investigated in an animal study. The study provided the timeconcentration curve in the CSF following administration of a free dexamethason solution or a suspension of solid-lipid nanoparticles (SLN) [56]. The study has shown a 13-fold increase in total dose in the CSF following delivery of dexamethason-loaded SLN [43]. This may indicate that solid-lipid nanoparticles, a nano-colloidal carrier, enhances drugs delivery from the inner ear to the CSF via the narrow passage of the cochlear aqueduct. The cochlear aqueduct is often filled with meshwork of loose tissue which resists movement of fluids. The surface active layer reagents (lecithin, poloxamer) in solid-lipid nanoparticles is known to facilitate transport via tight restrictions such as the cochlear aqueduct [44]. An animal study has shown that the inner ear can be used as a drug delivery [19] [41] route to the cerebrospinal fluid. The perilymphatic fluids in the compartment of the inner ear are connected to the cerebrospinal fluid in the subarachnoid space of the brain via the passage of the cochlear aqueduct. It has been shown that in a live animal, there exists a physiological flow from the compartment of the inner ear to the CSF in the subarachnoid space of the brain. A study that investigated the potential use of the inner ear as a delivery route to the CSF has shown that drugs effectively pass from the round-window membrane (RWM) of the inner ear to the CSF following a single trans-tympanic injection. The present invention provides a minimally invasive drug delivery means for prolonged administration to the CSF via the round-window membrane of the inner ear. The methods and systems described herein allow for improved surgical ease relative to intracerebroventricular (ICV) or intrathecal method. The present invention is directed to methods and systems for drug delivery to the cerebrospinal fluid (CSF) via inner ear. More 
specifically, the invention directed to a mimmally invasive method for prolonged delivery of brain-targeted therapeutics to the CSF via the perilymphatic fluid of the inner ear.

The perilymphatic fluid inside the inner ear and the cerebrospinal fluid (CSF) surrounding the central nervous system (CNS) are fluidly connected to one another via the passage of the cochlear aqueduct. Animal studies have shown that the perilymphatic fluid physiologically flows from the inner ear to the CSF in the subarachnoid compartment of the brain. An animal study that investigated the feasibly of using the inner ear as a delivery route to the CSF has shown that dexamethason acetate loaded to solid-lipid nanoparticles [2] (SLN) effectively transports to the CSF following trans-tympanic injection. While dexamethason acetate has long halflife in the CSF, most brain-targeted therapeutics requires frequent readrnimstration which is impractical to realize by trans-tympanic injection. The present invention provides a minimally invasive drug delivery system and method which overcomes this limitation. The delivery system is configured for prolonged administration of brain-targeted therapeutics to the CSF via the auditory ear canal. The system may be used for treatment of chronic CNS diseases and disorders, including pain. The delivery system is used for prolonged administration of fluid composition to the perilymphatic fluid of the inner ear. The system comprises of an implantable otological conduit which extends from the auditory ear canal to the round-window membrane, the conduit receives fluid from a nozzle in the auditory ear canal and deliver said fluid to the round-window membrane. The delivery system further comprising a dispenser which includes a dispensing nozzle connected to a fluid cartridge and an electronic module, the dispensing nozzle is placed inside the auditory canal in a closed proximity to the otological conduit. The dispensing nozzle operates intermittently to supply said fluid composition to the otological conduit based on a pre-programrned setting.Employing aspects of the delivery system described herein, a method for treating cllronic pain is described. The method comprising filling fluid analgesics into a cartridge which is connected to a dispensing nozzle. The analgesics are selected from a group consisting of analgesics opioid or 012-adrenergic agonist or a combination thereof. The method further includes implanting an otological conduit in the tympanic membrane for delivery of fluid from the auditory ear canal to the round window membrane, placing said dispensing nozzle inside the ear canal near the otological conduit and dispensing the fluid composition from the cartridge to the otological conduit at a predetermined delivery rate, such that the fluid composition perfuse through the round-window membrane to the perilymphatic fluid.Conveniently, the dispensing device of the present invention may be worn on the external ear as a removable hearing aid product, on any part of the body or on a clothing article. The dispensing device operates intermittently to supply fluid composition to the otological conduit inside the ear canal. The otological conduit conveys fluid to the site of the round-window membrane. Subsequently, fluid passes through the RWM by perfusion to the perilymphatic fluid inside the inner ear. The physiological flow of the perilymphatic fluid to the cerebrospinal fluid (CSF) carries the drug to subaracllr1oid space of brain and the dynamic flow within the subaracllrloid space distributes the drug in the brain's compartments.

\section{Conclusion}

The article reviews the feasibility of delivering drugs to brain via ear, and provides a novel route for delivering drugs to the brain tissues. The targeted delivery has been shown to reduce the toxicity and increase the therapeutic efficacy many-fold. The potential drugs for the treatment of most brain diseases are therefore often not able to cross these barriers. As a result, various drug delivery and targeting strategies are currently being developed to enhance the transport and distribution of drugs into the brain. Intra-cochlear administration shows great potential and offers a promising alternative to brain-targeted drug delivery.

\section{Acknowledgements}

The preparation of this review article was supervised and supported by Prof. (Dr.) N.V. Satheesh Madhav (D.I.T. University, Faculty of Pharmacy Mussorie Diversion Road Villmakkwalapo Bhagwantpur Dehradun, India) He is my Ph.D. supervisor for this research work. I express the gratitude for giving me supervision for such work. I also thank to Ms. Swarnima Pandey (Asst. Professor, A.C.P.R. Lucknow) for supporting me in preparation of article on such novel drug delivery.

\section{References}

[1]. Thierry Mom 1, Jean Chazal 2, Jean Gabrillargues 3, Laurent Gilain 1, Paul Avan 4 ), (Cochlear blood supply: an update on anatomy and function, Fr ORL - $2005 ; 88: 82$.

[2]. Active targeting of brain tumors using nanocarriers Arnaud Be'duneau, Patrick Saulnier, Jean-Pierre Benoit, Biomaterials , 28 (2007) 4947-4967

[3]. Development of inner ear afferent connections: forming primary neurons and connecting them to the developing sensory epithelia,Bernd Fritzsch, Brain Research Bulletin 60 (2003) 423-433.D

[4]. Yan Chen, Lihong Liu, Modern methods for delivery of drugs across the blood-brain barrier, Advanced Drug Delivery Reviews 64 (2012) 640-665. 
[5]. E. Neuwelt, N.J. Abbott, L. Abrey, W.A. Banks, B. Blakley, T. Davis, B. Engelhardt, P. Grammas, M. Nedergaard, J. Nutt, W. Pardridge, G.A. Rosenberg, Q. Smith, L.R. Drewes, Strategies to advance translational research into brain barriers, Lancet Neurol. 7 (2008) 84-96.

[6]. W.M. Pardridge, Blood-brain barrier drug targeting: the future of brain drug development, Mol. Interv. 3 (2003) 90-105 151.

[7]. Rip, G.J. Schenk, A.G. de Boer, Differential receptor-mediated drug targeting to the diseased brain, Expert Opin. Drug Deliv. 6 (2009) 227-237.

[8]. S.A. Pathan, Z. Iqbal, S.M. Zaidi, S. Talegaonkar, D. Vohra, G.K. Jain, A. Azeem, N. Jain, J.R. Lalani, R.K. Khar, F.J. Ahmad, CNS drug delivery systems: novel approaches, Recent Pat. Drug Deliv. Formul. 3 (2009) 71-89.

[9]. M. Ramsauer, J. Kunz, D. Krause, R. Dermietzel, Regulation of a blood-brain barrier-specific enzyme expressed by cerebral pericytes (pericytic aminopeptidase N/pAPN) under cell culture conditions, J. Cereb. Blood Flow Metab. 18 (1998) 1270-1281.

[10]. H. Wekerle, Immune protection of the brain-efficient and delicate, J. Infect. Dis.n186 (Suppl 2) (2002) S140-S144.

[11]. W.M. Pardridge, Molecular biology of the blood-brain barrier, Mol. Biotechnol. 30 (2005) 57-70.

[12]. N.J. Abbott, I.A. Romero, Transporting therapeutics across the blood-brain barrier,Mol. Med. Today 2 (1996) 106-113.

[13]. G. Lee, S. Dallas, M. Hong, R. Bendayan, Drug transporters in the central nervous system: brain barriers and brain parenchyma considerations, Pharmacol. Rev. 53(2001) 569-596.

[14]. E.M. Kemper, W. Boogerd, I. Thuis, J.H. Beijnen, O. van Tellingen, Modulation ofthe blood-brain barrier in oncology: therapeutic opportunities for the treatment of brain tumours? Cancer Treat. Rev. 30 (2004) 415-423. DEV

[15]. M. Intakhab Alama, Sarwar Bega, Abdus Samadb, Sanjula Babootaa,*, Kanchan Kohlia, Javed Ali a,Alka Ahujac, M. Akbard,Strategy for effective brain drug delivery, European Journal of Pharmaceutical Sciences 40 (2010) 385-403.

[16]. Erin E. Leary Swan, Mark J. Mescher, William F. Sewell, Sarah L. Tao, Jeffrey T. Borenstein, Inner Ear Drug Delivery for Auditory Applications, Copyright 2008 Elsevier B.V. Published in Advanced Drug Delivery Reviews, Vol. 60, No. 15, December 2008, pp. 1583-1599.

[17]. Ivri, yehuda (newport beach, ca, us), intracochlear drug delivery to the central nervous system united states patent application 20110208161 kind code: al application number:12/855152 publication date:08/25/2011 filing date:08/12/2010 .

[18]. Shulman A, Strashun AM. Fluid dynamics vascular theory of brain and inner-ear function in traumatic brain injury: a translational hypothesis for diagnosis and treatment, Int Tinnitus J. 2009;15(2):119-29.

[19]. Drug Delivery in Central Nervous System Diseases - Technologies, Markets and Companies Jain PharmaBiotech, February 2013, Pages: 349

[20]. Preliminary study on brain-targeted drug delivery via inner ear CHEN Gang; HOU Shi-xiang; HU Ping;JIN Miao-zhen;LIU Jun , Acta Pharmaceutica Sinica 0513-4870,2007,Issue 10 page:1102-1106.

[21]. Pardridge, W.M., Peptide drug delivery to the brain. Raven Press, New York, U.S.A., 1991.

[22]. Begley, D.J., The blood-brain barrier: principles for targeting peptides and drugs to the central nervous system. J Pharm Pharmacol, 48:136-146, 1996.

[23]. Formidable barriers: strategies: technological challenges;-targeted drug delivery to brain, ajhansee mishra, alok kumar dash, ijprd, 2012; vol 4(05): july-2012 (035 - 042).

[24]. Inner ear drug delivery for auditory application Serin e. leary swan a,b, mark j. mescher a, william f. sewell c,d,e, sarah 1 . tao a, jeffrey t. borenstein, advanced drug delivery reviews 60 (2008) 1583-1599.

.. This paper provides information about inner ear drug delivery for auditory application

[25]. N.V. Satheesh Madhav, Niharika Chauhan, Dehradun uttaranchal india, : a novel resealed erythrocytes loaded with repaglinide bionanoparticles, india, patent application publication , application no.3206/de1/2011 a , date of filing of application :11/11/2011, publication date : 17/05/2013.

[26]. N. V. Satheesh Madhav, Priyanka Bhatt, Dehradun uttaranchal india, : : a novel bio self nano -emulsifying drug delivery system (snedds) ofzidovudine, india, patent application publication, application no.3206/del/2011 a , date of filing of application $: 11 / 11 / 2011$, publication date : 17/05/2013. This paper provides information about biopolymers isolation and their role in drug delivery.

[27]. N.V. Satheesh Madhav, Nanuja Nautiyal, dehradun uttaranchal india, a novel bioplymer from fried seeds of amaranthus spinosus and its pharmaceutical applications, india, patent application publication, application no.3206/del/2011 a ,date of filing of application :11/11/2011, publication date : 17/05/2013.

[28]. N.V. Satheesh Madhav, Abhijeet Ohja, Nisha Poharswan, dehradun uttaranchal india, a novel composition for formulating biomontelukast microcapsules, india, patent application publication, application no.3206/del/2011 a ,date of filing of application $: 11 / 11 / 2011$, publication date : $17 / 05 / 2013$.

[29]. N.V. Satheesh Madhav, Abhay Pratap Yadav, Anupama Thapliyal, dehradun uttaranchal india, a novel biopolymer from the dry fruits of pistachia vera, india, patent application publication, application no.3206/del/2011 a date of filing of application $: 11 / 11 / 2011$, publication date : $17 / 05 / 2013$.

[30]. N.V. Satheesh Madhav, Akhilesh Vikram Singh, Divya Gupta, dehradun uttaranchal india, a novel biopolymer from fruit pulp of ficus carica and its novelistic pharmaceutical application, india, patent application publication, application no.3206/del/2011 a ,date of filing of application :11/11/2011, publication date : 17/05/2013.

[31]. N.V. Satheesh Madhav, Abhijeet Ojha, Amit Saini, a novel minoxidil bioniosomes using santalum album polymer as a biovesicle forming agent, india, patent application publication, application no.3206/del/2011 a, date of filing of application :11/11/2011, publication date : 17/05/2013.

[32]. Sevika Ramola, N.V. Satheesh Madhav, a novel formulation of emtricitabine solid lipid nanoparticles using a bioretardant from mangifera indica seeds, , india, patent application publication, application no.3206/del/2011 a ,date of filing of application $: 11 / 11 / 2011$, publication date : $17 / 05 / 2013$.

[33]. D.A. Borkholder State-of-the-art mechanisms of intracochlear drug delivery Curr Opin Otolaryngol Head Neck Surg, 16 (2008), pp. $472-477$

[34]. J.T. Borenstein Intracochlear drug delivery systems Expert Opin Drug Deliv, 8 (2011), pp. 1161-1174

[35]. T. Nakagawa, J. Ito Local drug delivery to the inner ear using biodegradable materials Ther Deliv, 2 (2011), pp. 807-814.

[36]. I. Pyykko, J. Zou, W. Zhang, Y. Zhang Nanoparticle-based delivery for the treatment of inner ear disorders Curr Opin Otolaryngol Head Neck Surg, 19 (2011), pp. 388-396.

[37]. E.E. Pararas, D.A. Borkholder, J.T. Borenstein Microsystems technologies for drug delivery to the inner ear Adv Drug Deliv Rev, 64 (2012), pp. 1650-1660.

[38]. K. Jahnke Permeability barriers of the inner ear. Fine structure and function Fortschr Med, 98 (1980), pp. 330-336.

[39]. S.K. Juhn Barrier systems in the inner ear Acta Otolaryngol Suppl, 458 (1988), pp. 79-83

[40]. R.T. Richardson, F. Noushi, S. O’LearyInner ear therapy for neural preservation Audiol Neurootol, 11 (2006), pp. 343-356 
[41]. A.N. Salt, S.K. Plontke Local inner-ear drug delivery and pharmacokinetics Drug Discov Today, 10 (2005), pp. 1299-1306

[42]. C.S. Kim, T.K. Cho, T.H. Jinn Permeability of the round window membrane to horseradish peroxidase in experimental otitis media Otolaryngol Head Neck Surg, 103 (1990), pp. 918-925

[43]. S.S. Chandrasekhar, R.Y. Rubinstein, J.A. Kwartler, M. Gatz, P.E. Connelly, E. Huang et al. Dexamethasone pharmacokinetics in the inner ear: comparison of route of administration and use of facilitating agents Otolaryngol Head Neck Surg, 122 (2000), pp. 521-528.

[44]. AN Salt Pharmacokinetics of Drug Entry into Cochlear Fluids The Volta Review, Volume 105(3) (monograph), 277-298.

[45]. Mosnier, I., D. Bouccara, O. Sterkers, "Management of IdiopathicSudden Sensorineural Hearing Loss," Otorhinolaryngol Nova, Vol. 9, 1999, pp. 217-223.

[46]. Hoffmann, K.K. and H. Silverstein, "Inner Ear Perfusion: Indications and Applications," Curr Opin Otolaryngol Head Neck Surg, Vol. 11, 2003, pp. 334-339.

[47]. Jackson, L.E. and H. Silverstein, "Chemical Perfusion of the Inner Ear" Otolaryngol Clin North Am, Vol. 35, 2002 , pp. 639-653.

[48]. Chandrasekhar, S.S., "Intratympanic Dexamethasone for Sudden Sensorineural Hearing Loss: Clinical and Laboratory Evaluation," Otol Neurotol, Vol. 22, 2001, pp. 18-23.

[49]. Plontke, S.K., H. Lowenheim, S. Preyer, P. Leins, K. Dietz, A. Koitschev, R. Zimmermann, H. -P. Zenner, "Outcomes Research Analysis of Continuous Intratympanic Glucocorticoid Delivery in Patients with Acute Severe to Profound Hearing Loss: Basis for Planning Randomized Controlled Trials," Acta Otolaryngol, Vol. 125, 2005, pp. 830-839.

[50]. Kopke, R.D., M.E. Hoffer, D. Wester, M.J. O'Leary, R.L. Jackson, "Targeted Topical Steroid Therapy in Sudden Sensorineural Hearing Loss," Otol Neurotol, Vol. 22, 2001, pp. 475-479.

[51]. Plaza, G. and C. Herraiz, "Intratympanic Steroids for Treatment of Sudden Hearing Loss after Failure of Intravenous Therapy," Otolaryngol Head Neck Surg, Vol. 137, 2007, pp. 74-78.

[52]. Gianoli, G.J. and J.C. Li, "Transtympanic Steroids for Treatment of Sudden Hearing Loss," Otolaryngol Head Neck Surg, Vol. 125, 2001, pp. 142-146.

[53]. Dallan, I., L. Bruschini, A. Nacci, P. Bruschini, C. Traino, F. Rognini, B. Fattori, "Transtympanic Steroids as a Salvage Therapy in Sudden Hearing Loss: Preliminary Results," ORL J Otorhinolaryngol Relat Spec, Vol. 68, 2005, pp. 247-252.

[54]. Rauch, S., "Intratympanic Steroids for Sensorineural Hearing Loss," Otolaryngol Clin North Am, Vol. 37, 2004, pp. $1061-1074$.

[55]. Haynes, D.S., M. O'Malley, S. Cohen, K. Watford, R.F. Labadie, "Intratympanic Dexamethasone for Sudden Sensorineural Hearing Loss after Failure of Systemic Therapy," Laryngoscope, Vol. 117, 2007, pp. 3-15.

[56]. Battista, R., "Intratympanic Dexamethasone for Profound Idiopathic Sudden Sensorineural Hearing Loss," Otolaryngol Head Neck Surg, Vol. 132, 2005, pp. 902-905.

[57]. Ryan, A.F., J.P. Harris, E.M. Keithley, "Immune-Mediated HearingLoss: Basic Mechanisms and Options for Therapy," Acta Otolaryngol Suppl, Vol. 548, 2002, pp. 38-43.

[58]. Yang, G.S.Y., H. Song, E.M. Keithley, J.P. Harris, "Intratympanic Immunosuppressives for Prevention of Immune-Mediated Sensorineural Hearing Loss," Am J Otol, Vol. 21, 2000, pp. 499-504.

[59]. E.L. Matteson, D.A. Fabry, S.E. Strome, C.L.W. Driscoll, C.W. Beatty, T.J. McDonald, "Autoimmune Inner Ear Disease: Diagnostic and Therapeutic Approaches in a Multidisciplinary Setting," J Am Acad Audiol, Vol. 14, 2003, pp. $225-230$.

[60]. Richardson, R.T., F. Noushi, S. O'Leary, "Inner Ear Therapy for Neural Preservation," Audiol Neurootol, Vol. 11, 2006, pp. 343356.

[61]. Beck, D.L., "Hair Cells: Review, Regeneration and Protection," Oticon Clinical Update, Vol. 1, 2007, p. 14.

[62]. Kuang, R. G. Hever, G. Zajic, O. Yan, F. Collins, J.C. Louis, E. Keithley, E. Magal, "Glial Cell Line-Derived Neurotrophic Factor. Potential for Otoprotection," Annals of the New York Academy of Sciences, 1999.

[63]. Lopez, I., V. Honrubia, S.C. Lee, W.H. Chung, L. Gang, K. Beykirch, P. Micevych, "The Protective Effect of Brain-Derived Neurotrophic Factor after Gentamicin Ototoxicity," The American Journal of Otology, Vol. 20, 1999, pp. $317-324$.

[64]. Oestreicher, E., M. Knipper, A. Arnold, H.P. Zenner, D. Felix, "Neurotrophin 3 Potentiates Glutamatergic Responses of IHC Afferents in the Cochlea In Vivo," European Journal of Neuroscience, Vol. 12, 2000, pp. 1584-1590.

[65]. Gillespie, L.N., G.M. Clark, M. Phillip, "Delayed Neurotrophin Treatment Supports Auditory Neuron Survival in Deaf Guinea Pigs," Neuroreport, Vol. 15, 2004, pp. 1121-1125.

[66]. Gluekert, R., Mario, J.M. Miller, Y. Zhu, D.M. Prieskorn, R.A. Altschuler, A. Schrott Fischer, "Deafferentiation-Associated Changes in Afferent and Effert Processes in the Guinea Pig Cochlea and Afferent Regeneration with Chronic Intrascalar BrainDerived Neurotrophic Factor and Acidic Fibroblast Growth Factor," The Journal of Comparative Neurology, Vol. 507, 2008, pp. $1602-1621$.

[67]. Miller, J.M., C.G. Le Prell, D.M. Prieskorn, N.L. Wys, R.A. Altschuler, "Delayed Neurotrophin Treatment Following Deafness Rescues Spiral Ganglion Cells from Death and Promotes Regrowth of Auditory Nerve Peripheral Processes: Effects of BrainDerived Neurotrophic Factor and Fibroblast Growth Factor," J Neurosci Res, Vol. 85, 2007, pp. 1959-1969.

[68]. Descamps L, Dehouck M-P, Torpier G, Cecchelli R. Receptormediated transcytosis of transferrin through blood-brain barrier endothelial cells. Am J Physiol Heart Circ Physiol 1996; 270(4 39-4).

[69]. Ocrant I. Insulin-like growth factor binding proteins in the nervous system. Adv Exp Med Biol 1991;293:471-82.

[70]. Wiessner JH, Hwang KJ. Binding of insulin to the external surface of liposomes. Effect of surface curvature, temperature, and lipid composition. Biochim Biophys Acta 1982;689(3):490-8.

[71]. Wiessner JH, Mar H, Baskin DG, Hwang KJ. Peptide-carrier interaction: induction of liposome fusion and aggregation by insulin. J Pharm Sci 1986;75(3):259-63.

[72]. Daniels TR, Delgado T, Helguera G, Penichet ML. The transferring receptor part II: targeted delivery of therapeutic agents in to cancer cells. Clin Immunol 2006;121(2):159-76.

[73]. Kang YS, Terasaki T, Ohnishi T, Tsuji A. In vivo and in vitro evidence for a common carrier mediated transport of choline and basic drugs through the blood-brain barrier. J Pharmacobiodyn 1990;13(6):353-60.

[74]. Tedeschi G, Lundbom N, Raman R, Bonavita S, Duyn JH, Alger JR, et al. Increased choline signal coinciding with malignant degeneration of cerebral gliomas: a serial proton magnetic resonancespectroscopy imaging study. J Neurosurg 1997;87(4):516-24.

[75]. Fenart L, Casanova A, Dehouck B, Duhem C, Slupek S, Cecchelli R, et al. Evaluation of effect of charge and lipid coating on ability of 60-nm nanoparticles to cross an in vitro model of the blood-brain barrier. J Pharmacol Exp Ther 1999;291(3):1017-22.

[76]. Lockman PR, Oyewumi MO, Koziara JM, Roder KE, Mumper RJ, Allen DD. Brain uptake of thiamine-coated nanoparticles. J Control Release 2003;93(3):271-82.

[77]. Kayser O, Lemke A, Hernandez-Trejo N. The impact of nanobiotechnology on the development of new drug delivery systems. Curr Pharm Biotechnol 2005;6:3-5.

[78]. Sahoo SK, Parveen S, Panda JJ. The present and future of nanotechnology in human health care. Nanomedicine 2007;3:20-31. 
[79]. Sahoo SK, Labhasetwar V. Nanotech approaches to drug delivery and imaging. Drug Discov Today 2003;8:1112-20.

[80]. Moghimi SM, Hunter AC, Murray JC. Nanomedicine: current status and future prospects. Faseb J 2005;19:311-30.

[81]. Torchilin VP. Drug targeting. Eur J Pharm Sci 2000;11(Suppl 2): S81-91.

[82]. Maeda H, Wu J, Sawa T, Matsumura Y, Hori K. Tumor vascular permeability and the EPR effect in macromolecular therapeutics: a review. J Control Release 2000;65:271-84.

[83]. Matsumura Y, Maeda H. A new concept for macromolecular therapeutics in cancer chemotherapy: mechanism of tumoritropic accumulation of proteins and the antitumor agent smancs. Cancer Res 1986;46:6387-92.

[84]. Choi $\mathrm{CH}$, Alabi CA, Webster P, Davis ME. Mechanism of active targeting in solid tumors with transferrin-containing gold nanoparticles. Proc Natl Acad Sci U S A 2010;107:1235-40.

[85]. Kocbek P, Obermajer N, Cegnar M, Kos J, Kristl J. Targeting cancer cells using PLGA nanoparticles surface modified with monoclonal antibody. J Control Release 2007;120:18-26.

[86]. Tong R, Yala L, Fan TM, Cheng J. The formulation of aptamer-coated paclitaxel-polylactide nanoconjugates and their targeting to cancer cells. Biomaterials 2010;31:3043-53.

[87]. Barraud L, Merle P, Soma E, Lefrançois L, Guerret S, Chevallier M, et al. Increase of doxorubicin sensitivity by doxorubicinloading into nanoparticles for hepatocellular carcinoma cells in vitro and in vivo. J Hepatol 2005;42:736-43.

[88]. Cheng FY, Su CH, Wu PC, Yeh CS. Multifunctional polymeric nanoparticles for combined chemotherapeutic and near-infrared photothermal cancer therapy in vitro and in vivo. Chem Commun (Camb) 2010;46:3167-9.

[89]. Chithrani DB, Jelveh S, Jalali F, van Prooijen M, Allen C, Bristow RG, et al. Gold nanoparticles as radiation sensitizers in cancer therapy. Radiat Res 2010;173:719-28.

[90]. de Boer, A.G. and Gaillard, P.J., In vitro models of blood-brain barrie: when to use which, Curr Med Chem, 2:203-209, 2002.

[91]. Mertsch, K. and Maas, J., Blood-brain barrier penetration and drug development from an industrial point of view, Curr Med Chem, 2:189-209, 2002.

[92]. Buchwald, P. and Bodor, N., Computer-aided drug design: the role of quantitative structure-property, structure- activity and structure-metabolism relationships (QSPR, QSAR, QSMR), Drugs Future, 27:577-588, 2002.

[93]. Kerns, E.H., Hightroughput physicochemical profiling for drug discovery. J Pharm Sci, 90:1838-1858, 2001.

[94]. Kakee, A., Terasaki, T. and Sugiyama, Y., Brain efflux index as a novel method of solute analyzing efflux transport at the bloodbrain barrier. J Pharmacol Exp Ther,277:1550-1559, 1996.

[95]. Begley, D.J., Squires, L.K., Zlokovic, B.V., Mitrovic, D.M., Hughes, C.C.W., Revest, P.A. and Greenwood, J., Permeability of the blood-brain barrier to the immunosuppressive cyclic peptide cyclosporine A. J Neurochem ,55:1222- 1230, 1998. 